\title{
Vortex-Line Condensation in Three Dimensions: A Physical Mechanism for Bosonic Topological Insulators
}

\author{
Peng Ye and Zheng-Cheng Gu \\ Perimeter Institute for Theoretical Physics, Waterloo, Ontario, Canada N2L 2 Y5
}

(Received 3 December 2014; published 19 June 2015)

\begin{abstract}
Bosonic topological insulators (BTIs) in three dimensions are symmetry-protected topological phases protected by time-reversal and boson number conservation symmetries. BTIs in three dimensions were first proposed and classified by the group cohomology theory, which suggests two distinct root states, each carrying a $\mathbb{Z}_{2}$ index. Soon after, surface anomalous topological orders were proposed to identify different root states of BTIs, which even leads to a new BTI root state beyond the group cohomology classification. In this paper, we propose a universal physical mechanism via vortex-line condensation from a 3D superfluid to achieve all three root states. It naturally produces a bulk topological quantum field theory description for each root state. Topologically ordered states on the surface are rigorously derived by placing topological quantum field theory on an open manifold, which allows us to explicitly demonstrate the bulk-boundary correspondence. Finally, we generalize the mechanism to $Z_{N}$ symmetries and discuss potential symmetry-protected topological phases beyond the group cohomology classification.

DOI: 10.1103/PhysRevX.5.021029

Subject Areas: Condensed Matter Physics,

Strongly Correlated Materials,

Topological Insulators
\end{abstract}

\section{INTRODUCTION}

Symmetry-protected topological states (SPTs) in strongly interacting bosonic and spin systems have been studied intensively recently [1-5]. By definition, the bulk of a SPT state only supports gapped bosonic excitations, but its boundary may exhibit anomalous quantum phenomena protected by global symmetry. As such, the usual threedimensional fermionic topological insulators (FTIs) [6-12] can be literally viewed as a fermionic SPT state [13]. A Haldane spin chain, which was proposed decades ago, is a typical example of SPT states in 1D [1,14-18]. Mathematically, given both spatial dimension and symmetry group $G$ as input data, one can apply the "group cohomology theory with $\mathbb{R} / \mathbb{Z}$ coefficient" to systematically classify SPT states [2]. Another mathematical tool, "cobordism," has also been applied, and some nontrivial SPT states beyond group cohomology theory have been proposed recently $[19,20]$. In addition to the above classification frameworks, a surge of broad interest has been shown from different approaches [22-54].

In this paper, we study bulk topological quantum field theory (TQFT) of bosonic topological insulators (BTIs) via the so-called "vortex-line condensation" mechanism. As a bosonic analog of the well-known FTIs, the so-called "bosonic topological insulators" were proposed first based

Published by the American Physical Society under the terms of the Creative Commons Attribution 3.0 License. Further distribution of this work must maintain attribution to the author(s) and the published article's title, journal citation, and DOI. on the group cohomology theory [2]. By definition, a BTI state is a nontrivial SPT state protected by $\mathrm{U}(1) \rtimes Z_{2}^{T}$ symmetry in three dimensions. Here, U(1) symmetry denotes the conservation of boson number, while timereversal symmetry $Z_{2}^{T}$ acts on bosons as $\mathcal{T}^{2}=1$ in the bulk. In the framework of group cohomology theory, such SPT states are classified by $\mathbb{Z}_{2} \times \mathbb{Z}_{2}[2,37,55]$. In other words, in comparison with the single $\mathbb{Z}_{2}$ index in FTIs of free fermions [6-12] which corresponds to the even or odd number of Dirac cones on the surface, there are two independent $\mathbb{Z}_{2}$ indices to label distinct BTI states and each index allows us to define a so-called "BTI root state" [55]. It was nicely proposed in Ref. [47] that the surface of a BTI supports topological order that (i) respects symmetry and (ii) cannot be realized on a 2D plane alone unless symmetry is broken. In the following, we briefly review all BTI root states.

The nontrivial phenomena of the first BTI root state can be characterized by its surface $Z_{2}$ topological order, where both $e$ and $m$ quasiparticles carry half-charge. In addition, if $Z_{2}^{T}$ is explicitly broken on such a surface and the bulk is fabricated in a slab geometry, one may expect a nontrivial electromagnetic response featured by a quantum Hall effect with odd-quantized Hall conductance on the surface and bulk Witten effect with $\Theta=2 \pi \bmod 4 \pi[47,49,56-58]$, which is different from $\Theta=\pi \bmod 2 \pi$ in FTI states of free fermions [59]. BTIs labeled by this $\mathbb{Z}_{2}$ index have been studied in detail via fermionic projective construction and dyon condensation [49]. The physical signature of the second BTI root state is characterized by its surface $Z_{2}$ topological order, where both $e$ and $m$ quasiparticles are Kramers doublets. Surprisingly, it has only recently been 
known that there is a new $\mathbb{Z}_{2}$ index that is beyond group cohomology classification $[19,20,47,48]$. As the third BTI root state, it supports a nontrivial surface with the so-called "all-fermion" $Z_{2}$ topological order, where all three nontrivial quasiparticles are self-fermionic and mutual semionic. Remarkably, an exactly solvable lattice model for this BTI has been proposed via the so-called Walker-Wang approach $[45,60]$, which confirms the existence of the third $\mathbb{Z}_{2}$ index.

\section{OVERVIEW}

Despite much progress in diagnosing surface phenomena of BTI states, throughout the paper, we stress that a welldefined bulk theory and bulk definition of symmetry are very crucial towards a controllable understanding of the surface quantum states. This concern is also highlighted in the conclusion section of Ref. [58]. If the bulk theory is unknown, the uniqueness of a proposed surface state is generically unclear. More concretely, one may understand the importance of a bulk definition through the following two aspects. First, given a 2D state that cannot be symmetrically realized in any $2 \mathrm{D}$ lattice model, it does not necessarily mean that the state can be realized on the surface of a 3D SPT phase. Second, when a surface phase transition occurs, the bulk does not necessarily experience a bulk phase transition, implying that a many-to-one correspondence between boundary and bulk is generically possible. Incidentally, a many-to-one correspondence was studied in quantum Hall states with high Landau levels [61,62]. In this paper, we also show that the first BTI root state (Sec. VI) exhibits a many-to-one correspondence. It generalizes the aforementioned descriptions of surface topological order where only $Z_{2}$ topological order is allowed.

One way to derive bulk field theory is the so-called hydrodynamical approach that we apply in this paper. We introduce this approach by briefly reviewing its application in fractional quantum Hall effect (FQHE). FQHE is a strongly correlated many-body quantum system, and it is technically hard to derive the bulk low-energy field theory by directly performing renormalization group analysis. However, one may apply the hydrodynamical approach whose main principle is to study collective modes of Hall systems at low energies. Since the bulk is gapped, it is sufficient to take quantum fluctuations of density $\rho$ and current $\vec{j}$ into account. Along this line of thinking, the bulk Chern-Simons theory is obtained, which encodes various "topological data" of the incompressible Hall state, such as modular $\mathcal{S}_{m}$ and $\mathcal{T}_{m}$ matrices, chiral central charge $c^{-}$, and other properties of edge conformal field theory [63-70]. Very recently, TQFT protected by global symmetry has also been studied intensively. For example, Lu and Vishwanath [24] imposed global symmetry to Chern-Simons theory and successfully classified some 2D SPT states protected by Abelian symmetry group. The success of such a hydrodynamical approach in 2D SPT [24] motivates us to develop a "universal hydrodynamical approach" for SPT phases in $3 \mathrm{D}$.

It is intricate to tackle bulk TQFT of all SPT states in 3D, which is far beyond the scope of the present work. In this paper, we restrict our attention to investigating the dynamic topological quantum field theory of aforementioned BTI states through considering exotic vortex-line condensations, which is pictorially illustrated in Fig. 1. Here, vortex lines mean the configuration of topological line defects in 3D superfluid states, e.g., helium-4. Such a vortex-line condensate state is shown to be described by a topological action in the form of

$$
S_{\mathrm{top}}=i \frac{K^{I J}}{2 \pi} \int b^{I} \wedge d a^{J}+i \frac{\Lambda^{I J}}{4 \pi} \int b^{I} \wedge b^{J},
$$

where $a^{I}$ are usual 1-form $\mathrm{U}(1)$ gauge fields and $b^{I}$ are 2-form U(1) gauge fields [71]. $K$ and $\Lambda$ are some $N \times N$ integer matrices that we elaborate on in the main text, $I, J=1,2, \ldots, N$. Surprisingly, we find that such a simple physical picture is sufficient to produce all three root states of 3D BTIs. More concretely, we find that the first two BTI root states within group cohomology classification can be achieved through a pure $b \wedge d a$-type term where symmetry transformations [either $Z_{2}^{T}$ or $\mathrm{U}(1)$ ] are defined in an

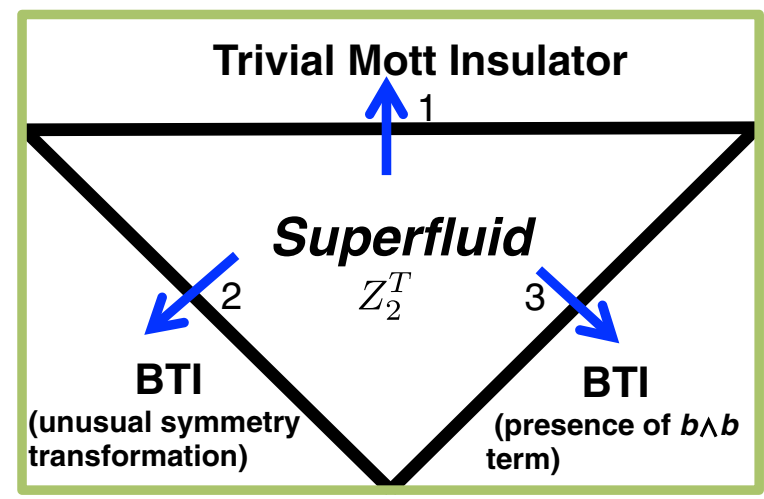

FIG. 1. Phases obtained by vortex-line condensation. In the phase transition 1, U(1) symmetry (i.e., boson number conservation) is restored from superfluid to a trivial Mott insulator by condensing strings (i.e., $2 \pi$ vortex lines). Thus, the trivial Mott insulator phase is formed by vortex-line condensation with $b \wedge d a$-type bulk field theory description. In the phase transition 2 , strings are also condensed and the bulk field theory is also $b \wedge d a$ type (see Secs. VI and VII), but the resultant Mott phase is a nontrivial SPT state [i.e., bosononic topological insulator (BTI)] since either $\mathrm{U}(1)$ or $Z_{2}^{T}$ symmetry transformation is defined in an unusual way. Thus, we end up with two different BTI root states. In the phase transition 3, strings are condensed in the presence of a nontrivial linking Berry phase term, or more precisely, a nontrivial multicomponent $b \wedge b$-type term. The nontrivial Mott phase is a BTI phase obtained in Sec. V, which is a SPT root state beyond group cohomology classification and supports "all-fermion" $Z_{2}$ surface topological order. Here, $Z_{2}^{T}$ denotes time-reversal symmetry with $\mathcal{T}^{2}=1$. 


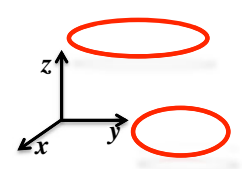

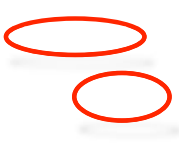

(b)

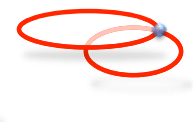

(c)

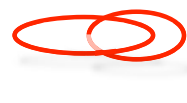

(d)
FIG. 2. Physical meaning of $b \wedge b$ topological term. In (a) the larger loop denotes a vortex line that is static and located on the $x y$ plane. The smaller loop is perpendicular to the $x y$ plane, parallel to the $y z$ plane, and moves toward the $z$ direction. In (b), two loops get closer to each other than (a). In (c), two loops collide with each other. The blue dot in (c) denotes the intersection of two loops. In (d), two loops are eventually linked to each other. The presence of $b \wedge b$ term will contribute a phase through this unlinking-linking process.

unusual way. The third BTI root state beyond group cohomology classification requires the presence of a nontrivial $b \wedge b$ term. As shown in Fig. 2, the topological term $b \wedge b$ contributes a $\mathrm{U}(1)$ phase to path-integral once two loops change the mutual linking number via collision. A brief summary is given in Table I. Our physical approach to 3D BTIs avoids the complication of advanced mathematical topics like cohomology theory and cobordism theory. And we believe that this physical picture sheds light on a more challenging question in the future: how to design microscopic interaction terms that can realize those proposed BTI states.

The vortex-line condensation picture is also formally generalized to some other symmetry groups, e.g., the unitary $Z_{N}$ group as discussed in Sec. VIII. It turns out that there are more potentially nontrivial SPT states beyond the group cohomology classification in 3D. The bulk dynamical quantum field theory of the new $Z_{N}$ SPT state is described by a single-component version of Eq. (1) with nonvanishing $\Lambda$. Based on our results, we conjecture the following.

Conjecture 1.-All SPT phases in 3D described by a $b \wedge b$ term are beyond the group cohomology classification.
The remainder of the paper is organized as follows. Section III is devoted to understanding the microscopic origins of bulk dynamical TQFT for 3D BTIs through the hydrodynamical approach. In this section, we start with a superfluid state in 3D and derive the TQFT description of the vortex-line condensate. In Sec. IV, some useful properties of the TQFT are studied when symmetry is not taken into account. By taking symmetry into account, the BTI state beyond group cohomology classification [20] is obtained in Sec. V where a nontrivial presence of the $b \wedge b$ term plays an important role. In Secs. VI and VII, the remaining two BTI root states within group cohomology classification are obtained via a pure $b \wedge d a$-type term by defining either $Z_{2}^{T}$ or U(1) symmetry transformation in an unusual way. In Sec. VIII, we show that a $b \wedge b$ term might also lead to $Z_{N}$ SPT phases beyond the group cohomology class. A concluding remark is made in Sec. IX, and some future directions are also proposed.

\section{HYDRODYNAMICAL APPROACH TO TOPOLOGICAL QUANTUM FIELD THEORY}

\section{A. 3D superfluid state and its dual description}

The exotic states discussed in this paper are built from a well-known parent state: the 3D superfluid (SF) state described by

$$
\mathcal{L}=\frac{\rho}{2}\left(\partial_{\mu} \theta\right)^{2}
$$

at low energies. Here, $\rho$ is the superfluid density and $\theta$ is the $\mathrm{U}(1)$ phase angle of the superfluid. The spatial gradient of $\theta$ costs energy such that a spatially uniform value of $\theta$ is picked up in the ground state, rendering a spontaneous symmetry breaking of the global U(1) symmetry group (i.e., the particle number conservation of bosons). In order to capture the periodicity of $\theta$ in the continuum field theory, we express $\theta$ in terms of a smooth part and a singular part:

TABLE I. A brief summary of main results in Secs. V-VII. As in the 1D chiral Luttinger liquid theory, which can be derived by putting Chern-Simons action on a 2D disk with a 1D boundary, the surface of each BTI root state is also rigorously derived by putting the bulk field theory on an open 3D manifold with a 2D boundary. Symmetry transformations [both U(1) and time reversal $Z_{2}^{T}$ ] are rigorously defined in the bulk. The first and second BTI root states are within the group cohomology classification and obtained by the changing definition of either U(1) symmetry or $Z_{2}^{T}$ symmetry in the bulk. The third BTI root state is beyond group cohomology and its realization requires an addition of "cosmological constant term" $b \wedge b$ term. More details (e.g., the integer matrices $K^{I J}, \Lambda^{I J}$ ) are present in the main text.

\begin{tabular}{lcc}
\hline \hline BTI & Bulk TQFT & Surface topological order \\
\hline $\begin{array}{l}\text { The first BTI root state in } \\
\text { Sec. VI }\end{array}$ & $\left(K^{I J} / 4 \pi\right) b_{\mu \nu}^{I} \partial_{\lambda} a_{\rho}^{J} \epsilon^{\mu \nu \lambda \rho}$ & $\begin{array}{c}Z_{p}(p=\text { even) topological order with exotic electric } \\
\text { charge assignment. U(1) is defined in an unusual way. }\end{array}$ \\
$\begin{array}{l}\text { The second BTI root } \\
\text { state in Sec. VII }\end{array}$ & $\left(K^{I J} / 4 \pi\right) b_{\mu \nu}^{I} \partial_{\lambda} a_{\rho}^{J} \epsilon^{\mu \nu \lambda \rho}$ & $Z_{2} \begin{array}{c}\text { topological order where both } e \text { and } m \text { carry Kramers } \\
\text { doublets. } Z_{2}^{T} \text { is defined in an unusual way. }\end{array}$ \\
$\begin{array}{l}\text { The third BTI root state } \\
\text { in Sec. V (beyond } \\
\text { cohomology) }\end{array}$ & $\left(K^{I J} / 4 \pi\right) b_{\mu \nu}^{I} \partial_{\lambda} a_{\rho}^{J} \epsilon^{\mu \nu \lambda \rho}+\left(\Lambda^{I J} / 16 \pi\right) b_{\mu \nu}^{I} b_{\lambda \rho}^{J} \epsilon^{\mu \nu \lambda \rho \rho}$ & \begin{tabular}{c}
$Z_{2} \begin{array}{c}\text { topological order where all } e, m \text {, and } \epsilon \text { quasiparticles } \\
\text { are fermionic. } \mathrm{U}(1) \rtimes Z_{2}^{T} \text { is defined in a usual way. }\end{array}$ \\
\hline \hline
\end{tabular} \\
\hline
\end{tabular}


$\theta=\theta^{s}+\theta^{v}$. By substituting this $\theta$ decomposition into Eq. (2) and introducing a Hubbard-Stratonovich auxiliary vector field $\mathcal{J}^{\mu}$ [72-74], one may express Eq. (2) in the following equivalent formalism:

$$
\mathcal{L}=\frac{1}{2 \rho}\left(\mathcal{J}_{\mu}\right)^{2}+i \mathcal{J}^{\mu}\left(\partial_{\mu} \theta^{s}+\partial_{\mu} \theta^{v}\right),
$$

which goes back to Eq. (2) once $\mathcal{J}^{\mu}$ is integrated out. It is obvious that $\mathcal{J}^{\mu}$ can be interpreted as a supercurrent of the 3D SF state. Integrating out $\theta^{s}$ leads to a constraint $\delta\left(\partial_{\mu} \mathcal{J}^{\mu}\right)$ in the path-integral measure. This constraint can be resolved by introducing a 2-form noncompact $\mathrm{U}$ (1) gauge field $b_{\mu \nu}: \mathcal{J}^{\mu} \underline{\underline{\operatorname{def}}}(1 / 4 \pi) \epsilon^{\mu \nu \lambda \rho} \partial_{\nu} b_{\lambda \rho}$. Both the physical quantity $\mathcal{J}^{\mu}$ and the Lagrangian Eq. (3) are invariant under the usual smooth gauge transformation:

$$
b_{\mu \nu} \rightarrow b_{\mu \nu}+\partial_{[\mu} \xi_{\nu]}
$$

where $\xi_{\mu}$ is a smooth 4 -vector. $\partial_{[\mu} \xi_{\nu]}$ stands for $\partial_{\mu} \xi_{\nu}-\partial_{\nu} \xi_{\mu}$. Eventually, the Lagrangian Eq. (3) is transformed to the following gauge theory:

$$
\mathcal{L}=\frac{1}{48 \pi^{2} \rho} h^{\mu \nu \lambda} h_{\mu \nu \lambda}+\frac{i}{2} b_{\mu \nu} \Sigma^{\mu \nu},
$$

where the field strength $h_{\mu \nu \lambda}$ is a rank-3 antisymmetric

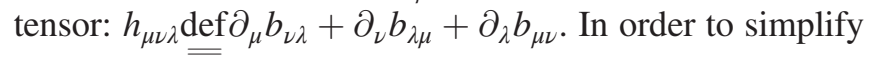
notation, $\mathcal{L}_{h}$ is introduced via

$$
\mathcal{L}_{h} \stackrel{\text { def }}{=} \frac{1}{48 \pi^{2} \rho} h^{\mu \nu \lambda} h_{\mu \nu \lambda}
$$

which is the Maxwell term of the 2-form U(1) gauge field $b_{\mu \nu}$. The vortex-line (i.e., string) current operator $\Sigma_{\mu \nu}$, which is antisymmetric, is defined through the singular $\theta^{v}$ :

$$
\Sigma^{\mu \nu} \stackrel{\operatorname{def}}{=} \frac{1}{2 \pi} \epsilon^{\mu \nu \lambda \rho} \partial_{\lambda} \partial_{\rho} \theta^{v}
$$

which is generically nonzero for nontrivial homotopy mapping. The gauge transformation shown above automatically ensures that there is a continuity equation for $\Sigma_{\mu \nu}$, i.e., $\partial_{\nu} \Sigma^{\mu \nu}=0$. Hereafter, the nouns strings, vortex lines, and closed loops are used interchangeably. The vortex-line configuration is very dilute in superfluid. The factor $\frac{1}{2}$ in the coupling term $\frac{1}{2} b_{\mu \nu} \Sigma^{\mu \nu}$ naturally arises as a standard convention for the antisymmetric tensor field coupling in $3+1 \mathrm{D}$ space-time.

\section{B. Trivial Mott insulators realized by condensing vortex lines (strings)}

Considering strong correlation effects (like Hubbard interactions), we expect that, passing through a critical point where the tension of vortex lines decreases to zero, the string configuration (denoted by the path-integral measure $\mathcal{D} \Sigma$ ) will be proliferated energetically. In other words, vortex-line condensation [75,76] sets in. The pathintegral formalism of vortex-line condensation was given in Refs. [76-78]. Here, we do not go into technical details but briefly review the basic method. A single string can be described by reparametrization-invariant Nambu-Goto action. A wave function $\Psi$ can be introduced in quantum theory of strings. Similar to the usual quantum theory of particles, after promoting the quantum mechanics of single string to field theory of many strings, $\Psi$ will be viewed as the creation operator (in operator formalism) or the quantum amplitude (in path-integral formalism) of a given string configuration.

In condensate of bosons, the ground state is formed by equal-weight superposition of all kinds of boson configurations in real space, which leads to a macroscopic wave function. The amplitude fluctuation of the condensate order parameter is gapped, but the phase fluctuation $\theta$ is gapless and governed by Eq. (2). Likewise, once vortex-line condensation sets in, all vortex-line configurations have the same quantum amplitude $\Psi$. In contrast to condensate of bosons, the $\mathrm{U}(1)$ phase of $\Psi$ of vortex-line condensate is given by a Wilson line $e^{i \int d x_{\mu} \Theta^{\mu}}$ and governed by the Lagrangian

$$
\mathcal{L}=\frac{1}{2} \phi_{0}^{2}\left(\partial_{[\mu} \Theta_{\nu]}-b_{\mu \nu}\right)^{2}+\mathcal{L}_{h},
$$

where the antisymmetrization symbol is defined as usual: $\partial_{[\mu} \Theta_{\nu]}$ def $\partial_{\mu} \Theta_{\nu}-\partial_{\nu} \Theta_{\mu} \cdot\left|\phi_{0}\right|^{2}$ is the "phase stiffness" of the vortex-line condensate. The presence of dynamical gauge field $b_{\mu \nu}$ gaps out the gapless phase fluctuation from $\Theta_{\mu}$. One may split the phase vector into the smooth part $\Theta_{\mu}^{s}$ and the singular part $\Theta_{\mu}^{v}: \Theta_{\mu}=\Theta_{\mu}^{s}+\Theta_{\mu}^{v}$, where $\int d^{3} \mathbf{r}(1 / 4 \pi) \nabla \cdot \nabla \times \boldsymbol{\Theta}^{v} \in \mathbb{Z}$. Therefore, the gauge group of $b_{\mu \nu}$ is compactified by absorbing $\Theta_{\mu}^{v}$. Note that, in the dual Lagrangian Eq. (4) in the SF phase, $b_{\mu \nu}$ is not compact.

Based on Eq. (7), we may formally perform duality transformation in this vortex-line condensation to obtain a $b \wedge d a$ term where $a$ is 1 -form gauge field. For this purpose, we introduce a Hubbard-Stratonovich auxiliary tensor field $\Sigma_{\mu \nu}$ :

$$
\mathcal{L}=i \frac{1}{2} \Sigma_{\mu \nu}\left(\partial^{[\mu} \Theta^{\nu]}-b^{\mu \nu}\right)+\mathcal{L}_{h}+\frac{1}{8 \phi_{0}^{2}} \Sigma_{\mu \nu} \Sigma^{\mu \nu},
$$

where the physical interpretation of $\Sigma_{\mu \nu}$ is the same as the one defined in Eqs. (4) and (6).

Integrating over $\Theta_{\mu}^{s}$ in Eq. (8) yields a constraint $\delta\left(\partial_{\nu} \Sigma^{\mu \nu}\right)$ in the path-integral measure. This constraint can be resolved by introducing a 1-form noncompact $\mathrm{U}(1)$ gauge field $a_{\mu}$ : 


$$
\Sigma^{\mu \nu} \underline{\underline{\operatorname{def}}}-\frac{1}{2 \pi} \epsilon^{\mu \nu \lambda \rho} \partial_{\lambda} a_{\rho}
$$

indicating that $a_{\mu}$ field strength is physically identified as the "supercurrent" $\Sigma_{\mu \nu}$ of vortex lines. The vector field $a_{\mu}$ is a gauge field since under the gauge transformation,

$$
a_{\mu} \rightarrow a_{\mu}+\partial_{\mu} \eta,
$$

the physical observable $\Sigma^{\mu \nu}$ is invariant. $a_{\mu}$ takes values smoothly on the whole real axis so that $a_{\mu}$ is noncompact, thereby, leading to $\partial_{\nu} \Sigma^{\mu \nu}=0$. Then, the dual formalism of Lagrangian Eq. (7) is given by

$$
\begin{aligned}
\mathcal{L}= & i \frac{1}{4 \pi} a_{\mu} \epsilon^{\mu \nu \lambda \rho} \partial_{\nu} b_{\lambda \rho}+i a_{\mu} j_{v}^{\mu}+\mathcal{L}_{h} \\
& +\frac{1}{64 \pi^{2} \phi_{0}^{2}} f_{\mu \nu} f^{\mu \nu} .
\end{aligned}
$$

The field strength tensor $f_{\mu \nu} \underline{\underline{\operatorname{def}}} \partial_{[\mu} a_{\nu]}$ as usual. The monopole current of the string condensate is given by $\left.j_{v}^{\mu}=-(1 / 4 \pi) \partial_{\nu} \partial_{[\lambda} \Theta_{\rho]}^{v}\right]^{\mu \nu \lambda \rho}$. We may redefine $b$ by absorbing $d \Theta^{v}: b_{\mu \nu}-\partial_{[\mu} \Theta_{\nu]}^{v} \rightarrow b_{\mu \nu}$. After removing the irrelevant Maxwell terms $\mathcal{L}_{h}$ and $f_{\mu \nu} f^{\mu \nu}$ at low energies, we end up with the following topological $B F$ Lagrangian:

$$
\mathcal{L}=i \frac{1}{4 \pi} \epsilon^{\mu \nu \lambda \rho} b_{\mu \nu} \partial_{\lambda} a_{\rho}
$$

As expected, the coefficient $1 / 4 \pi$ of the first term in Eq. (12) indicates that there is no ground-state degeneracy (GSD) [79-85] on a 3-torus $\mathbb{T}^{\nVdash}$ [86-88]. In this sense, the bulk state has no intrinsic topological order [79-85]. In terms of exterior products, the term can be rewritten as $(1 / 2 \pi) b \wedge d a$, where $d a \equiv f$ is a 2-form field strength tensor.

\section{Adding a vortex line (string) linking Berry phase term into the trivial Mott insulator}

Here, we attempt to explore the possibility of nontrivial Mott insulators. To begin, we add a topological Berry phase term into Eq. (7) to describe a potential nontrivial topological vortex-line condensate:

$$
\begin{aligned}
\mathcal{L}= & \frac{1}{2} \phi_{0}^{2}\left(\partial_{[\mu} \Theta_{\nu]}^{s}-b_{\mu \nu}\right)^{2}+\mathcal{L}_{h} \\
& -i \frac{\Lambda}{16 \pi} \epsilon^{\mu \nu \lambda \rho}\left(\partial_{[\mu} \Theta_{\nu]}^{s}-b_{\mu \nu}\right)\left(\partial_{[\lambda} \Theta_{\rho]}^{s}-b_{\lambda \rho}\right),
\end{aligned}
$$

where $b$ is redefined by absorbing $d \Theta^{v}$ : $b_{\mu \nu}-\partial_{[\mu} \Theta_{\nu]}^{v} \rightarrow b_{\mu \nu}$. By applying the duality transformation, this topological vortex-line condensate can be equivalently described by the following "BF + BB" TQFT:

$$
\mathcal{L}_{\text {top }}=i \frac{1}{4 \pi} \epsilon^{\mu \nu \lambda \rho} b_{\mu \nu} \partial_{\lambda} a_{\rho}+i \frac{\Lambda}{16 \pi} \epsilon^{\mu \nu \lambda \rho} b_{\mu \nu} b_{\lambda \rho} .
$$

In detail, in Eq. (13), the term $\sim d \Theta^{s} \wedge d \Theta^{s}$ is a total derivative term and can be neglected. Then, by introducing a Hubbard-Stratonovich auxiliary tensor field $\Xi^{\mu \nu}$ (antisymmetric), Eq. (13) is transformed to

$$
\begin{aligned}
\mathcal{L}= & i \frac{1}{2} \Xi^{\mu \nu}\left(\partial_{[\mu} \Theta_{\nu]}^{s}-b^{\mu \nu}\right)+\frac{1}{8 \phi_{0}^{2}} \Xi_{\mu \nu} \Xi^{\mu \nu} \\
& +i \frac{\Lambda}{8 \pi} \epsilon^{\mu \nu \lambda \rho} \partial_{[\mu} \Theta_{\nu]}^{s} b_{\lambda \rho}-i \frac{\Lambda}{16 \pi} \epsilon^{\mu \nu \lambda \rho} b_{\mu \nu} b_{\lambda \rho}+\mathcal{L}_{h} \\
= & i \Theta_{\mu}^{s} \partial_{\nu}\left(\Xi^{\mu \nu}+\frac{\Lambda}{4 \pi} \epsilon^{\mu \nu \lambda \rho} b_{\lambda \rho}\right)-i \frac{1}{2} \Xi_{\mu \nu} b^{\mu \nu} \\
& -i \frac{\Lambda}{16 \pi} \epsilon^{\mu \nu \lambda \rho} b_{\mu \nu} b_{\lambda \rho}+\frac{1}{8 \phi_{0}^{2}} \Xi_{\mu \nu} \Xi^{\mu \nu}+\mathcal{L}_{h} .
\end{aligned}
$$

Integrating out $\Theta_{\mu}^{s}$ leads to the conservation constraint, $\partial_{\nu}\left(\Xi^{\mu \nu}+(\Lambda / 4 \pi) \epsilon^{\mu \nu \lambda \rho} b_{\lambda \rho}\right)=0$, which can be resolved by introducing a 1 -form noncompact U(1) gauge field $a_{\mu}$ :

$$
\Xi^{\mu \nu} \stackrel{\mathrm{def}}{=}-\frac{1}{2 \pi} \epsilon^{\mu \nu \lambda \rho} \partial_{\lambda} a_{\rho}-\frac{\Lambda}{4 \pi} \epsilon^{\mu \nu \lambda \rho} b_{\lambda \rho} .
$$

This is a modified version of Eq. (9), where the $b \wedge b$ term is absent. Plugging this expression into the second term in Eq. (15) yields the topologically invariant Lagrangian Eq. (14).

In Eq. (14), only topological terms are preserved. $\mathcal{L}_{h}$, which is defined in Eq. (5), is the Maxwell kinetic term of $b_{\mu \nu}$ with scaling dimension more irrelevant than the two topological terms in Eq. (14). In addition, we consider the phase region that is deep in the string condensation phase and far away from the phase boundary between SF and string condensate. As such, the "phase stiffness" $\left|\phi_{0}\right|^{2} \rightarrow \infty$ is taken. The first term in Eq. (14) is the standard $b \wedge d a$ term that already exists in Sec. III B. What is new here is the second term, $b \wedge b$. It was previously introduced in mathematical physics [89]. It is also applied to loop quantum gravity [90] with cosmological constant. Its physical meaning is pictorially shown in Fig. 2. The above topologically invariant Lagrangian is gauge invariant under the following gauge transformations:

$$
b_{\mu \nu} \rightarrow b_{\mu \nu}+\partial_{[\mu} \xi_{\nu]}, \quad a_{\mu} \rightarrow a_{\mu}+\partial_{\mu} \eta-\Lambda \xi_{\mu} .
$$

Formally, the above single-component theory can be generalized into a multicomponent theory:

$$
\mathcal{L}_{\text {top }}=i \frac{K^{I J}}{4 \pi} \epsilon^{\mu \nu \lambda \rho} b_{\mu \nu}^{I} \partial_{\lambda} a_{\rho}^{J}+i \frac{\Lambda^{I J}}{16 \pi} \epsilon^{\mu \nu \lambda \rho} b_{\mu \nu}^{I} b_{\lambda \rho}^{J} .
$$

In terms of exterior products, the action (1) is obtained. Without loss of generality, it is sufficient to consider a 
symmetric matrix $\Lambda^{I J}$ and assume $K^{I J}$ to be an identity matrix of rank $N$, i.e.,

$$
K=\operatorname{diag}(1,1, \ldots, 1)_{N \times N}=\rrbracket,
$$

with $I, J=1,2, \ldots, N .\left\{a_{\mu}^{I}\right\}$ are noncompact 1-form U(1) gauge fields and $\left\{b_{\mu \nu}^{I}\right\}$ are compact 2-form $\mathrm{U}(1)$ gauge fields, respectively, as a straightforward generalization of the above one-component theory. The above topologically invariant Eq. (17) Lagrangian with the particular $K$ matrix Eq. (18) is the central result of this paper [its abstract form in terms of exterior products is given by Eq. (1)], and we use it to describe all BTI phases as well as some new $Z_{N}$ SPT phases. Physically, such a multicomponent theory can be viewed as a collection of many $3 \mathrm{D}$ trivial Mott insulators mutually entangled via a $\Lambda$ term. Hereafter, we also call indices $I, J, \ldots$ flavor indices.

\section{GENERAL PROPERTIES OF THE TOPOLOGICAL QUANTUM FIELD THEORY}

In this section, we study the multicomponent topological quantum field theory defined by Eq. (17) in a general setting without any global symmetry implementation. In this section as well as Sec. V, all analyses are done by implicitly assuming $K$ takes the form in Eq. (18) unless otherwise stated [e.g., the gauge transformation and $\mathbb{G} \mathbb{L}(N, \mathbb{Z})$ transformation in Sec. IVA are valid for general $K]$.

\section{A. Gauge transformation and bulk $\mathbb{G} \mathbb{L}(N, \mathbb{Z})$ transformation}

As aforementioned, the presence of the $b \wedge b$ term drastically changes the gauge structures. The gauge transformation of the multicomponent theory Eq. (17) is given by

$$
\begin{aligned}
b_{\mu \nu}^{I} & \rightarrow b_{\mu \nu}^{I}+\partial_{[\mu} \xi_{\nu]}^{I}, \\
a_{\mu}^{I} & \rightarrow a_{\mu}^{I}+\partial_{\mu} \eta^{I}-\left(K^{-1} \Lambda\right)^{I I^{\prime}} \xi_{\mu}^{I^{\prime}},
\end{aligned}
$$

which generalizes Eq. (16).

To see the gauge transformation more clearly, we reexpress the first two topological terms (denoted by $\mathcal{L}_{\text {top }}$ ) in Lagrangian Eq. (17) as

$$
\begin{aligned}
\mathcal{L}_{\text {top }}= & i \frac{\Lambda^{I J}}{32 \pi} \epsilon^{\mu \nu \lambda \rho}\left[b_{\mu \nu}^{I}+\left(\Lambda^{-1} K\right)^{I I^{\prime}} \partial_{[\mu} a_{\nu]}^{I^{\prime}}\right] \\
& \cdot\left[b_{\lambda \rho}^{J}+\left(\Lambda^{-1} K\right)^{J J^{\prime}} \partial_{[\lambda} a_{\rho]}^{J^{\prime}}\right] .
\end{aligned}
$$

From this expression, one may easily examine the correctness of Eq. (19). To obtain this equivalent expression, we apply the following two facts: (i) a closed space-time manifold is taken and (ii) $a_{\mu}$ is noncompact such that the term $\sim d a \wedge d a$ is a total derivative.
Then, we perform two independent general linear $(\mathbb{G L})$ transformations represented by matrices $W, M \in \mathbb{G} \mathbb{L}(N, \mathbb{Z})$, on gauge fields $b_{\mu \nu}^{I}$ and $a_{\mu}^{I}$, respectively. $\mathbb{G} \mathbb{L}$ transformations keep the quantization of gauge charges of both gauge fields unaffected:

$$
\underline{b}_{\mu \nu}^{I}=\left(W^{-1}\right)^{I J} b_{\mu \nu}^{J}, \quad \underline{a}_{\mu}^{I}=\left(M^{-1}\right)^{I J} a_{\mu}^{J},
$$

where $W, M$ are two $N \times N$ matrices with integer-valued entries and $|\operatorname{det} W|=|\operatorname{det} M|=1$. These transformations are nothing but a relabeling of the same low-energy physics. After the transformations, a new set of parameters $(\underline{K}, \underline{\Lambda})$ are introduced via

$$
\underline{K}=W^{T} K M, \quad \underline{\Lambda}=W^{T} \Lambda W,
$$

which leads to a new Lagrangian in the same form as Eq. (17). $W$ and $M$ are two independent $\mathbb{G L}$ transformations. In any basis, $|\operatorname{det} K|$ rather than $\operatorname{det} K$ is invariant. Therefore, our choice Eq. (18) is universal once bulk topological order is absent.

\section{B. Quantization conditions on path-integral field variables}

For simplicity, we merely consider the one-component theory. It is straightforward to generalize all of the results obtained below to multicomponent theory by adding component indices. The action is given by

$$
\begin{aligned}
S= & i \frac{1}{4 \pi} \int d^{4} x b_{\mu \nu} \partial_{\lambda} a_{\rho} \epsilon^{\mu \nu \lambda \rho} \\
& +i \frac{\Lambda}{16 \pi} \int d^{4} x b_{\mu \nu} b_{\lambda \rho} \epsilon^{\mu \nu \lambda \rho} .
\end{aligned}
$$

It is known that the classical action $S$ alone is not enough to define a quantum system. One must properly define the partition function $Z$ where the path-integral measure should be properly defined in addition to the classical action. First, a formal integration over the 1 -form gauge field $a_{\mu}$ leads to a flat connection constraint on the 2-form gauge field with local flatness $d b=0$. Second, we note that there is a shift in the microscopic justification of the single-component action based on the mechanism vortex-line condensation: $b_{\mu \nu}+\partial_{[\mu} \Theta_{\nu]}^{v} \rightarrow b_{\mu \nu}$, which leads to a quantization condition of $b_{\mu \nu}$. Quantitatively, we have the following quantization condition on $b$ on a closed 2D manifold $\mathcal{S}$ embedded in 4D space-time:

$$
\oiint_{\mathcal{S}} b=\oiint_{\mathcal{S}} d \Theta^{v}=\iiint_{\mathcal{V}} d d \Theta^{v}=2 \pi \times \text { integer }
$$

where $\mathcal{S}=\partial \mathcal{V}$. In deriving the third BTI root state (see Table I) that needs a nontrivial $b \wedge b$, we use this quantization condition which is obtained based on the 
microscopic origin vortex-line condensation. As a matter of fact, once the topological terms $\mathrm{BF}+\mathrm{BB}$ are formally derived from our microscopic origin, we may extend our discussion on the topological field theory to a more general background. In other words, this quantization condition on the $b$ field can be derived in a more mathematically rigorous way by formally introducing compactness of both gauge fields, which are fundamentally constrained by two conditions [i.e., Eqs. (A1) and (A2) given in Appendix A]. In Secs. VI and VII, where the derivation of the first and second BTI root states (see Table I) are presented, respectively, we continue to use this general method.

By using the condition Eq. (24), we derive the following periodic shift (more details are presented in Appendix B):

$$
\Lambda \rightarrow \Lambda+1 .
$$

Thus, $\Lambda$ is compactified to a finite region $[0,1)$. Further, one may consider a large gauge transformation, which leads to quantization of $\Lambda \in \mathbb{Z}$. The derivation of this result may be considered as a special case of Eq. (71) with $N=1$. Therefore, without time-reversal symmetry, any allowed nonzero $\Lambda$ is identified as $\Lambda=0$.

More generally, the above periodicity shift of the singlecomponent theory is also applicable to the diagonal entries $\Lambda^{I I}$ of a generic multicomponent theory. For off-diagonal entries, the results are still unchanged. For example, since the $\Lambda^{13}$ term and the $\Lambda^{31}$ term are equal to each other $\left(\Lambda^{13}=\Lambda^{31}\right)$, the total actions of the mixture of $b_{\mu \nu}^{1}$ and $b_{\mu \nu}^{3}$ are actually $2 \times\left(\Lambda^{13} / 16 \pi\right) \int d^{4} x \epsilon^{\mu \nu \lambda \rho} b_{\mu \nu}^{1} b_{\lambda \rho}^{3}$. We note that there are eight equivalent copies in Eq. (B1) where the two $b$ fields are the same. However, in the $\Lambda^{13}$ term where the two $b$ fields are different, there are only four equivalent copies. Overall, loss and gain are balanced such that the periodicity of $\Lambda^{13}$ is still 1 .

In addition, the condition Eq. (24) can also be applied to derive the surface theory. Because of this condition, $b^{I}$ is locally flat and a new 1-form compact gauge field $\tilde{a}_{\mu}^{I}$ for each index $I$ can be introduced via

$$
b_{\mu \nu}^{I} \underline{\underline{\operatorname{def}}} \partial_{[\mu} \tilde{a}_{\nu]}^{I}=\tilde{f}_{\mu \nu}^{I}
$$

where $\tilde{f}_{\mu \nu}^{I}$ is the field strength tensor of $\tilde{a}_{\mu}^{I}$. For the sake of convenience, $\mu, \nu, \lambda=0,1,2$ is implicitly assumed in all of the surface variables. The compactness of $\tilde{a}^{I}$ can be easily understood by substituting it into the quantization condition of $b^{I}$. Then, the magnetic flux of $\tilde{a}^{I}$ piercing a closed $\mathcal{S}$ is allowed to be nonzero, which means that monopoles of $\tilde{a}^{I}$ are allowed so that $\tilde{a}^{I}$ is compactified. After substituting this expression into the $b \wedge b$ term in Eq. (17), we end up with the following surface Lagrangian:

$$
\mathcal{L}_{\partial}=\frac{\Lambda^{I J}}{4 \pi} \epsilon^{\mu \nu \lambda} \tilde{a}_{\mu}^{I} \partial_{\nu} \tilde{a}_{\lambda}^{J} .
$$

\section{BOSONIC TOPOLOGICAL INSULATORS IN THE PRESENCE OF $b \wedge b$ TOPOLOGICAL TERM}

In this section, we implement symmetry and consider the $b \wedge b$ term and end up with the third BTI root state (the last row in Table I) that is beyond group cohomology classification.

\section{A. Definition of time-reversal transformation}

Given the microscopic origin of the topological quantum field theory Eq. (17) in Sec. III, we know that $b_{\mu \nu}$ is minimally coupled to vortex lines (i.e., strings), while $a_{\mu}$ is minimally coupled to bosonic particles. As a result, the time-reversal transformations of all gauge fields and excitations can be consistently defined in the following usual way (the spatial directions are denoted by $i=1,2,3$ ):

$$
\begin{array}{cl}
\mathcal{T} a_{0}^{I} \mathcal{T}^{-1}=a_{0}^{I}, & \mathcal{T} a_{i}^{I} \mathcal{T}^{-1}=-a_{i}^{I}, \\
\mathcal{T} j_{0} \mathcal{T}^{-1}=j_{0}, & \mathcal{T} j_{i} \mathcal{T}^{-1}=-j_{i}, \\
\mathcal{T} b_{0, i}^{I} \mathcal{T}^{-1}=-b_{0, i}^{I}, & \mathcal{T} b_{i, j}^{I} \mathcal{T}^{-1}=b_{i, j}^{I}, \\
\mathcal{T} \Sigma_{0, i} \mathcal{T}^{-1}=-\Sigma_{0, i}, & \mathcal{T} \Sigma_{i, j} \mathcal{T}^{-1}=\Sigma_{i, j},
\end{array}
$$

where every flavor transforms in the same way.

The pure $b \wedge f$ term (i.e., the $b \wedge b$ term is absent) in Eq. (17) is invariant under these transformation rules. The definition of time-reversal transformation in Eqs. (28) and (29) implies that a pure $b \wedge d a$ term necessarily leads to a trivial SPT state for the reason that bulk topological order is absent and symmetry transformation is defined in a usual way. The possibility of nontrivial SPT states arising from pure a $b \wedge d a$ term is discussed in Secs. VI and VII, where either $\mathrm{U}(1)$ or time-reversal symmetry has to be modified unusually, as shown in Fig. 1. Therefore, in this section, $\Lambda=0$ always results in a trivial SPT state, and a nontrivial SPT state requires a nontrivial $\Lambda$ matrix.

\section{B. Quantization condition implemented by time-reversal symmetry}

Under the time-reversal symmetry transformation Eq. (29), the $b \wedge b$ term is transformed to $-\left(\Lambda^{I J} / 16 \pi\right) \epsilon^{\mu \nu \lambda \rho} b_{\mu \nu}^{I} b_{\lambda \rho}^{J}$. Superficially, this sign change implies that the ground state of topological field theory labeled by $\Lambda$ always "breaks" time-reversal symmetry. However, we show that the periodicity shift of $\Lambda^{I J}$ provides a chance for restoring time-reversal symmetry. We derived a periodic shift Eq. (25) on $\Lambda$ where merely U(1) symmetry is considered. A new problem arises: Is Eq. (25) still valid in the presence of time-reversal symmetry?

We reconsider a simple one-component theory shown in Eq. (23). In the presence of time-reversal symmetry, the space-time manifold becomes unoriented such that topological response can be probed $[19,21]$. A simple 
understanding is that a normal vector at each space-time point changes sign under time-reversal symmetry such that a time-reversal invariant system requires that each spacetime point is directionless. In an unoriented space-time manifold, a $\pi$ cubic flux of a 2 -form gauge field $b_{\mu \nu}$ becomes the minimally allowed value. To have a physical picture for the flux quantization condition on an unoriented manifold, we consider the simplest case-a flux insertion process for a Möbius strip. Very different from a cylinder, where the inserted flux must be in units of $2 \pi$, the Möbius strip allows the inserted flux to be in units of $\pi$, namely, $\oint_{L} A_{\mu} d l_{\mu}=\pi \times$ integer, because a particle on a Möbius strip must pick up an even winding number to travel back to its origin. In this sense, if we still use the same notations as in Appendix B $1, \mathcal{N}_{0 x}$ and $\mathcal{N}_{y z}$ now are half-integers instead of integers. The last line in Eq. (B1) now equals $\frac{1}{2} \Lambda \pi \times$ integer, such that the periodicity of $\Lambda$ is now enhanced to

$$
\Lambda \rightarrow \Lambda+4
$$

We now move on to the off-diagonal entries, e.g., $\Lambda^{13}$ in a multicomponent theory. At present, there are $N$ components of topological vortex-line condensations, which, superficially, implies that there are $N \mathrm{U}(1)$ charge conservation symmetries. However, in our physical system, only one U(1) should be taken into account. Then, when we evaluate the sum of the $\Lambda^{13}$ and $\Lambda^{31}$ terms, either the $b^{1}$ gauge group or $b^{2}$ forms a $\pi$ cubic flux, not both. Therefore, the periodicity of $\Lambda^{13}$ is enhanced from 1 to 2 : i.e., $\Lambda^{13} \rightarrow \Lambda^{13}+2$. In summary, all of the above results indicate that $\Lambda^{I J}$ in the presence of time-reversal symmetry take the following values:

$$
\Lambda^{I I}=0, \pm 2, \quad \Lambda^{I J}=0, \pm 1 \quad(\text { for } I \neq J) .
$$

This quantization condition is protected by time-reversal symmetry.

We note that SPT states (including both trivial and nontrivial states) are defined by the following two common conditions: (i) bulk has no intrinsic topological order and (ii) the bulk state respects symmetry. Condition (i) is always satisfied in our construction since GSD $=1$, as shown in Sec. IVA, where $K=\square$. If $\Lambda$ entries are defined under the requirement of Eq. (31), condition (ii) is also satisfied. Thus, there are infinite number of $\Lambda$ matrices that satisfy Eq. (31) and can be viewed as SPT states with $\mathrm{U}(1) \mathrm{U}(1) \rtimes Z_{2}^{T}$ symmetry. But which are trivial and which are nontrivial? For example, is $\Lambda=2$ a trivial or a nontrivial SPT? In subsequent discussions, we aim to answer this question.

\section{Trivial SPT states with $|\operatorname{det} \Lambda|=1$}

All $\Lambda$ matrices ( $K=\rrbracket$ is implicit all the time) that satisfy the quantization conditions Eq. (31) are SPT states.
Generically, with an open boundary condition, the surface phenomena of SPT states are expected to capture information of triviality and nontriviality. Therefore, one may wonder what are nontrivial signatures of surface phenomena? Conceptually, one should first find the set of physical observables that describe the surface physics.

Definition 1: Surface physical observables.-The physical observables of Eq. (27) are composed by ground-state degeneracy, self-statistics, and mutual statistics of gapped quasiparticles. All of this information can be read out from modular $\mathcal{S}_{m}$ and $\mathcal{T}_{m}$ matrices. Since there is no further 1D boundary, chiral central charge $c^{-}$is not an observable on the surface. Notice that, for a topological phase defined on a 2D plane, the physical observables are $\mathcal{S}_{m}, \mathcal{T}_{m}$, and $c^{-}$.

Now our question is changed to, how can we use these physical observables to tell a nontrivial SPT from a trivial SPT? The essential physics is the so-called "obstruction" or "anomaly.'. More precisely, we define it as follows.

Definition 2: Obstruction (quantum anomaly).-By "obstruction," we mean that the set of physical observables (defined in Definition 1) of the surface theory cannot be reproduced on a $2 \mathrm{D}$ plane by any local bosonic lattice model with symmetry. Otherwise, the obstruction is free. Nontriviality of a SPT state corresponds to the presence of obstruction. Now, we are in the position to distinguish trivial and nontrivial SPT states. We first consider a subset of $\Lambda$ matrices that satisfy Eq. (31):

$$
|\operatorname{det} \Lambda|=1 \text {. }
$$

Mathematically, any $\Lambda$ matrix in this subset can be expressed in terms of two "fundamental blocks," namely, $\Lambda_{t 1}$ and $\Lambda_{t 2}$, given by the Cartan matrix of the $\mathrm{E}_{8}$ group [62,91-93]:

$$
\Lambda_{t 1}=\left(\begin{array}{ll}
0 & 1 \\
1 & 0
\end{array}\right), \quad \Lambda_{t 2}=\left(\begin{array}{cccccccc}
2 & 1 & 0 & 0 & 0 & 0 & 0 & 0 \\
1 & 2 & 1 & 0 & 0 & 0 & 0 & 0 \\
0 & 1 & 2 & 1 & 0 & 0 & 0 & 1 \\
0 & 0 & 1 & 2 & 1 & 0 & 0 & 0 \\
0 & 0 & 0 & 1 & 2 & 1 & 0 & 0 \\
0 & 0 & 0 & 0 & 1 & 2 & 1 & 0 \\
0 & 0 & 0 & 0 & 0 & 1 & 2 & 0 \\
0 & 0 & 1 & 0 & 0 & 0 & 0 & 2
\end{array}\right) \text {. }
$$

The subscript $t$ in $\Lambda_{t 1}$ and $\Lambda_{t 2}$ stands for trivial (which we explain below). One can show that all $\Lambda$ matrices that satisfy Eqs. (31) and (32) can be expressed as the following canonical form, namely, a direct sum of several $\Lambda_{t 1}$ and $\pm \Lambda_{t 2}$ up to an arbitrary $\mathbb{G} \mathbb{L}$ transformation:

$\Lambda=W^{T}\left(\Lambda_{t 1} \oplus \Lambda_{t 1} \oplus \cdots \oplus \pm \Lambda_{t 2} \oplus \pm \Lambda_{t 2} \oplus \cdots\right) W$. 
Since GL transformation $W$ does not affect physical observables, whether SPT states in this subset are trivial or not essentially depends on the properties of the two fundamental blocks.

$\Lambda_{t 1}$ gives a trivial SPT state (i.e., a trivial Mott insulator) since its surface physical observables are trivial gapped boson excitations and nothing else. Such surface states can be realized on a 2D lattice model with symmetry. According to Definition 2, the obstruction is free and, thus, the $3 \mathrm{D}$ bulk state is trivial.

In contrast to $\Lambda_{t 1}$ whose chiral central charge $c_{-}=0$, the very feature of $\Lambda_{t 2}$ is that it has an "irreducible" value of $c_{-}=8$, which plays the role of the "generator" of all trivial $\Lambda$ matrices that admit nonzero $c_{-}$. We call $\pm \Lambda_{t 2}$ " $c$ generators." By irreducible, we mean that one can prove that $c_{-}=8$ is the minimal absolute value of all $\Lambda$ matrices that satisfy Eqs. (31) and (32). Since $c_{-} \neq 0$, one may wonder if the $\Lambda_{t 2}$ surface state breaks time-reversal symmetry once it is laid on a $2 \mathrm{D}$ plane alone. To solve this puzzle, we should, again, focus attention on physical observables on the surface rather than the formal Lagrangian in Eq. (27). On the grounds that a surface is a $2 \mathrm{D}$ closed manifold by definition, there is no further $1 \mathrm{D}$ edge so that $c_{-}$is not detectable on the surface, which is also summarized in Definition 1. Thus, $\Lambda_{t 2}$ still gives a trivial SPT state.

Technically, the triviality of $\Lambda_{t 2}$ can also be understood by using the following Gauss-Milgram sum formula $[61,94,95]$ :

$$
\frac{1}{\sqrt{|\operatorname{det} \Lambda|}} \sum_{a} e^{i 2 \pi J_{a}}=e^{i 2 \pi i c^{-} / 8},
$$

for a bosonic Abelian topological phase defined on a 2D plane. Here, $a$ denotes quasparticles and $J_{a}$ is topological spin, which is determined by the modular $\mathcal{T}_{m}$ matrix. The diagonal entries $T_{m}^{a a}=e^{i 2 \pi J^{a}}$ in a quasiparticle basis. The chiral central charge $c^{-}$is determined only modulo 8 . We consider a $\Lambda_{t 2}$ surface state. According to Definition 1, the surface physical observables are determined by $\mathcal{S}_{m}$ and $\mathcal{T}_{m}$. The two modular matrices are given by $\mathcal{S}_{m}=1, \mathcal{T}_{m}=1$, both of which are just a number. Thus, the surface is nothing but a trivial gapped bosonic phase with timereversal symmetry and supporting only trivial identity particles. Then, one may wonder if the time-reversal symmetry can be preserved when we define such a set of physical observables on a $2 \mathrm{D}$ plane, because on a $2 \mathrm{D}$ plane we need to further consider chiral central charge, which is a signature of time-reversal breaking. The answer is yes. The reason is that all possible states on a $2 \mathrm{D}$ plane with the same $\mathcal{S}_{m}$ and same $\mathcal{T}_{m}$ have $c^{-}=0 \bmod 8$ due to Eq. (34). Since $c^{-}=0$ is a solution, a time-reversal symmetric state on a $2 \mathrm{D}$ plane is achievable without any difficulty. Thus, according to Definition $2, \Lambda_{t 2}$ labels a trivial SPT state.

\section{Trivial SPT states with $|\operatorname{det} \Lambda|>1$}

The above discussion leads to a set of trivial SPT states defined by Eqs. (31) and (32). All of these trivial states do not admit topological order on the surface. How about topologically ordered surface (i.e., $|\operatorname{det} \Lambda|>1$ )?

While the quantization conditions Eq. (31) guarantee that the bulk is symmetric, surface might break symmetry. If symmetry is manifestly broken on the surface, such a surface state can be realized on a 2D plane, which is free of obstruction from symmetry requirement. The corresponding bulk state is a trivial SPT state. Therefore, hereafter we merely focus on the symmetry-preserving surface state. But the question arises, how can we judge that the symmetry is preserved on the surface?

A symmetric surface governed by Eq. (27) with $\Lambda$ matrix must describe the same set of physical observables as those on the surface with a $-\Lambda$ matrix. In order to leave the surface physical observables unaffected under time reversal (i.e., $\Lambda \rightarrow-\Lambda$ ), one obvious way is to consider the following equivalence under $\mathbb{G L}$ transformation, namely, "GL equivalence":

$$
W^{T} \Lambda W=-\Lambda, \quad \exists W \in \mathbb{G} \mathbb{L}(N, \mathbb{Z}),
$$

which does not change physical observables in Definition 1. We introduce the following symbol

$$
\Lambda \stackrel{\underline{G L}}{\underline{\underline{L}}}-\Lambda
$$

to denote this equivalence relation. However, such a topological order has no obstruction if it is defined on a 2D plane with symmetry since such a $\mathbb{G} \mathbb{L}$ transformation can also be regularly performed on a $2 \mathrm{D}$ plane. Thus, the only way toward nontrivial SPT states is to find a new method such that (i) it can transform $\Lambda$ to $-\Lambda$ leaving all physical observables unaffected and (ii) it is forbidden to be regularly done on a $2 \mathrm{D}$ plane. This is what we do in Sec. V E where an "extended $\mathbb{G} \mathbb{L}$ transformation" Eq. (37) is defined.

Before proceeding further, we give some examples. The simplest one is $\Lambda=2$, which can be connected to $\Lambda=-2$ via neither Eq. (35) nor Eq. (37), so that the corresponding bulk state is a trivial SPT with a symmetry-breaking surface. Another example is $\Lambda=\left(\begin{array}{cc}2 & 0 \\ 0 & -2\end{array}\right)$, which can be connected to $\Lambda=\left(\begin{array}{cc}-2 & 0 \\ 0 & 2\end{array}\right)$ via Eq. (35), so that the corresponding bulk state is a trivial SPT with a symmetrypreserving surface.

\section{E. Extended $\mathbb{G} \mathbb{L}$ transformation and nontrivial SPT states}

In order to obtain nontrivial SPT states, again, we resort to the fact that the chiral central charge on the surface is not a physical observable as discussed in Sec. V C. We relax the $\mathbb{G L}$ transformation by arbitrarily adding fundamental 
blocks like $\Lambda_{t 1}$ and $\pm \Lambda_{t 2}$ defined in Sec. VC along the diagonal entries of the $\Lambda$ matrix. This stacking is legitimate since all fundamental blocks correspond to trivial SPT states, which do not induce phase transitions.

Technically, we perform a so-called extended $\mathbb{G} \mathbb{L}$ transformation via the following equivalence relation:

$$
\begin{aligned}
& W^{T}\left(\Lambda \oplus \Lambda_{t 1} \oplus \Lambda_{t 1} \cdots\right) W \\
& \quad=(-\Lambda) \oplus \pm \Lambda_{t 2} \oplus \pm \Lambda_{t 2} \cdots, \quad \exists W \in \mathbb{G} \mathbb{L}\left(N^{\prime}, \mathbb{Z}\right),
\end{aligned}
$$

which also leaves physical observables in Definition 1 unaffected. Here, the left-hand side contains $\left[\left(N^{\prime}-N\right) / 2\right] \Lambda_{t 1}$ matrices, while the right-hand side contains $\left[\left(N^{\prime}-N\right) / 8\right]$ " $\pm \Lambda_{t 2}$ " matrices. Here, $\Lambda$ is $N \times N$ as usual. The extended $\mathbb{G L}$ transformation for $\Lambda$ means that adding several fundamental blocks to both $\Lambda$ and $-\Lambda$ results in two new matrices; we can then connect these two new matrices, i.e., $(\Lambda \oplus$ $\left.\Lambda_{t 1} \oplus \Lambda_{t 1} \ldots\right)$ and $(-\Lambda) \oplus \pm \Lambda_{t 2} \oplus \pm \Lambda_{t 2} \ldots$, by performing a $\mathbb{G} \mathbb{L}$ transformation $W$.

Since $\left[\Lambda_{t 2} \oplus\left(-\Lambda_{t 2}\right)\right] \underset{\mathbb{G} L}{=} \sum^{8} \oplus \Lambda_{t 1}$, the \pm signs reduce to an overall sign, i.e.,

$$
\begin{aligned}
& W^{T}\left(\Lambda \oplus \Lambda_{t 1} \oplus \Lambda_{t 1} \ldots\right) W \\
& \quad=(-\Lambda) \oplus\left[ \pm\left(\Lambda_{t 2} \oplus \Lambda_{t 2} \ldots\right)\right], \quad \exists W \in \mathbb{G} \mathbb{L}\left(N^{\prime}, \mathbb{Z}\right) .
\end{aligned}
$$

If $\Lambda$ and $-\Lambda$ are connected to each other via an extended $\mathbb{G} \mathbb{L}$ transformation, we define the symbol

$$
\Lambda \underline{\underline{e \mathbb{G} \mathbb{L}}}-\Lambda
$$

to denote their equivalence relation.

We find that there is a unique solution that supports nontrivial SPT. It is the Cartan matrix of the $\mathrm{SO}(8)$ group, denoted by $\Lambda_{\mathrm{so} 8}[91]$ :

$$
\Lambda_{\mathrm{so} 8}=\left(\begin{array}{cccc}
2 & 1 & 1 & 1 \\
1 & 2 & 0 & 0 \\
1 & 0 & 2 & 0 \\
1 & 0 & 0 & 2
\end{array}\right) .
$$

More precisely, the following transformation exists:

$$
\begin{aligned}
& W^{T}\left(\Lambda_{\mathrm{so} 0} \sum^{4} \oplus \Lambda_{t 1}\right) W \\
& \quad=\left(-\Lambda_{\mathrm{s} 08}\right) \oplus \Lambda_{t 2}, \quad \exists W \in \mathbb{G} \mathbb{L}(12, \mathbb{Z}) .
\end{aligned}
$$

Instead of looking directly for the explicit matrix form of $W$, the existence of $W$ can be justified by checking the equivalence of the physical observables (see Definition 1) between $\left(\Lambda_{\mathrm{so} 8} \sum^{4} \oplus \Lambda_{t 1}\right)$-surface Chern-Simons theory and $\left(-\Lambda_{\mathrm{so} 8}\right) \oplus \Lambda_{t 2}$-surface Chern-Simons theory. Indeed, both share the same excitation spectrum that is formed by four distinct gapped quasiparticles, and they share the same real $\mathcal{S}_{m}$ and $\mathcal{T}_{m}$ matrices:

$$
\begin{aligned}
& \mathcal{S}_{m}=\left(\begin{array}{cccc}
1 & 1 & 1 & 1 \\
1 & 1 & -1 & -1 \\
1 & -1 & 1 & -1 \\
1 & -1 & -1 & 1
\end{array}\right), \\
& \mathcal{T}_{m}=\left(\begin{array}{cccc}
1 & 0 & 0 & 0 \\
0 & -1 & 0 & 0 \\
0 & 0 & -1 & 0 \\
0 & 0 & 0 & -1
\end{array}\right) .
\end{aligned}
$$

From the above $\mathcal{S}_{m}$ and $\mathcal{T}_{m}$, we read much information. In addition to the trivial boson excitations (identity quasiparticles), there are three distinct fermions denoted by $f_{1}, f_{2}, f_{3}$. Braiding $f_{i}$ around $f_{j}(\forall i, j, i \neq j)$ once leads to an Aharonov-Bohm phase $e^{i \pi}=-1$. Consequently, through the equivalence relation Eq. (37), we end up with a SPT state whose surface has topological order.

Furthermore, it is a nontrivial SPT, i.e., a bosonic topological insulator. To see its nontriviality clearly, one may attempt to look for obstruction defined in Definition 2. We put the set of physical observables (given by the modular $\mathcal{S}_{m}$ and $\mathcal{T}_{m}$ matrices of $\Lambda_{\mathrm{so} 8}$ ) on a $2 \mathrm{D}$ plane. According to the Gauss-Milgram sum formula Eq. (34), the chiral central charge $c^{-}=4 \bmod 8$. Therefore, all states on a 2D plane necessarily have nonzero chiral central charge, indicating that time-reversal symmetry is necessarily broken. Thus, such an obstruction defined in Definition 2 gives a 3D nontrivial SPT state labeled by $\Lambda_{\mathrm{so} 8}$.

To summarize, we derive a BTI state from our bulk field theory where a nontrivial multicomponent $b \wedge b$ term plays an essential role. We stress that this BTI state is obtained and examined rigorously from bulk to boundary step by step.

\section{BOSONIC TOPOLOGICAL INSULATORS FROM PURE $b \wedge d a$ TERM-(I)}

In the above discussions, we consider the time-reversal transformation defined by Eqs. (28) and (29) such that the pure $b \wedge d a$ term always describes trivial SPT states, i.e., trivial Mott insulators in Fig. 1. In the following, we show that the first BTI root state (the first row in Table I) can be obtained by pure a $b \wedge d a$ topological term where $\mathrm{U}(1)$ charge symmetry is defined in an unusual way.

\section{A. $\mathbb{Z}_{\mathbf{2}}$ nature of bulk $\mathrm{U}(1)$ symmetry definition}

The unusual $\mathrm{U}(1)$ symmetry transformation can be directly characterized by a $\Theta$ term $F \wedge F$ in the response theory, where $F_{\mu \nu}$ is the field strength of the external electromagnetic field $A_{\mu}$. Technically, one may start with a generic $b \wedge d a$ theory with $N$ components and then add an electromagnetic coupling term like $\sum_{I}\left(q_{1}^{I} / 4 \pi\right) F_{\mu \nu} \partial_{\lambda} a_{\rho}^{I} \epsilon^{\mu \nu \lambda \rho}+$ $\sum_{I}\left(q_{2}^{I} / 4 \pi\right) A_{\mu} \partial_{\nu} b_{\lambda \rho}^{I} \epsilon^{\mu \nu \lambda \rho}$, where $\left\{q_{1}^{I}\right\},\left\{q_{2}^{I}\right\}$ are two integral charge vectors. The first term is the coupling between face 
variable $F_{\mu \nu}$ and vortex-line current $(1 / 2 \pi) \partial_{\lambda} a_{\rho} \epsilon^{\mu \nu \lambda \rho}$, where the additional $\frac{1}{2}$ is due to the double counting of the pair indices $\mu, \nu$. The second term is the coupling between the link variable $A_{\mu}$ and the boson particle current $(1 / 4 \pi) \partial_{\nu} b_{\lambda \rho} \epsilon^{\mu \nu \lambda \rho}$.

We may expect an electromagnetic response action with the bulk $\Theta$ term in addition to the usual Maxwell terms:

$$
\mathcal{S}_{\text {EM response }}=\int d^{4} x \frac{\Theta}{32 \pi^{2}} F_{\mu \nu} F_{\lambda \rho} \epsilon^{\mu \nu \lambda \rho}+\cdots,
$$

where the centered dots denote Maxwell terms. For SPT states, there are only two choices: $\Theta=0 \bmod 4 \pi$ or $2 \pi \bmod 4 \pi$. This $\mathbb{Z}_{2}$ classification can be understood through alternative insights, such as the charge lattice of bulk quasiparticles [49] or the statistical Witten effect [58], both of which rely on the pioneering studies of dyon statistics in Ref. [57]. But the procedure of the integration over $a^{I}$ and $b^{I}$ is quite subtle in the presence of background gauge field $A$, since $b^{I}$ and $a^{I}$ are constrained by several conditions. In the next section, we give an $N=2$ simple example.

\section{B. Example with $N=\mathbf{2}$}

We start with a $b \wedge d a$ theory with the following $K$ matrix:

$$
K=\left(\begin{array}{ll}
0 & 1 \\
1 & 2
\end{array}\right)
$$

More explicitly, the total Lagrangian is given by

$$
\begin{aligned}
\int d^{4} x \mathcal{L}_{0}= & \int i \frac{1}{2 \pi} b^{1} \wedge d a^{2}+i \int \frac{1}{2 \pi} b^{2} \wedge d a^{1} \\
& +i \int \frac{2}{2 \pi} b^{2} \wedge d a^{2} .
\end{aligned}
$$

Time-reversal symmetry is defined in the usual way shown in Eqs. (28) and (29). All gauge fields are constrained by Eqs. (A1) and (A2)) (by adding indices $I, J, \ldots$ ).

Let us move on to the surface theory. Starting with Eq. (43), one can integrate out compact $a^{1}$ on an unoriented manifold, leading to the quantization condition on $b^{2}$ :

$$
\oiint_{\mathcal{S}} b^{2}=\pi \times \text { integer }
$$

which indicates that $b^{2}$ is locally flat and a new 1-form compact gauge field $\tilde{a}^{2}$ can be introduced in a way that is similar to Eq. (26):

$$
b_{\mu \nu}^{2} \underline{\underline{\operatorname{def}}} \partial_{[\mu} \tilde{a}_{\nu]}^{2} .
$$

Thus, by means of Eqs. (29), $\tilde{a}_{\mu}^{2}$ is transformed as a pseudovector under time-reversal symmetry: $\tilde{a}_{0}^{2} \rightarrow-\tilde{a}_{0}^{2}$, $\tilde{a}_{i}^{2} \rightarrow \tilde{a}_{i}^{2}, i=x, y, z$.
The term $\sim b^{2} \wedge d a^{2}$ in Eq. (43) provides a surface Chern-Simons term:

$$
\mathcal{L}_{\partial}=i \frac{1}{\pi} \epsilon^{\mu \nu \lambda} \tilde{a}_{\mu}^{2} \partial_{\nu} a_{\lambda}^{2},
$$

which can also be reformulated by introducing a matrix $K_{\partial} \underline{=} \operatorname{def}\left(\begin{array}{ll}0 & 2 \\ 2 & 0\end{array}\right)$ in the standard convention of $K$-matrix ChernSimons theory [63]. $a_{\mu}^{2}$ and $\tilde{a}_{\mu}^{2}$ form a two-dimensional vector $\left(a_{\mu}^{2}, \tilde{a}_{\mu}^{2}\right)^{T}$. The ground state of Eq. (46) supports a $Z_{2}$ topological order [96] associated with four gapped quasiparticle excitations $(1, e, m, \varepsilon)$. Here, " 1 " denotes identical particles. Both $e$ and $m$ are bosonic, while $\varepsilon$ is a fermion. The particle $e$ carries +1 gauge charge of $a_{\mu}^{2}$, while the particle $m$ carries +1 gauge charge of $\tilde{a}_{\mu}^{2} \cdot \varepsilon$ carries +1 gauge charges of both gauge fields. These three nontrivial quasiparticles all have mutual semionic statistics; i.e., full braiding one particle around another distinct particle leads to a $\pi$ Aharonov-Bohm phase.

To obtain BTI states, we expect the U(1) symmetry transformation may be performed in an unusual way. For this purpose, we add the following electromagnetic coupling term to $\mathcal{L}_{0}$ in Eq. (43):

$$
\frac{q_{1}}{4 \pi} F_{\mu \nu} \partial_{\lambda} a_{\rho}^{2} \epsilon^{\mu \nu \lambda \rho}+\frac{q_{2}}{4 \pi} A_{\mu} \partial_{\nu} b_{\lambda \rho}^{2} \epsilon^{\mu \nu \lambda \rho} .
$$

Let us consider that a surface is located on $z=0$ plane. The surface theory is described by Eq. (46). The coupling term contributes the following surface electromagnetic coupling terms:

$$
\frac{q_{1}}{2 \pi} \epsilon^{\mu \nu \lambda} A_{\mu} \partial_{\nu} a_{\lambda}^{2}+\frac{q_{2}}{2 \pi} \epsilon^{\mu \nu \lambda} A_{\mu} \partial_{\nu} \tilde{a}_{\lambda}^{2},
$$

where Eq. (45) is applied. Based on the Chern-Simons term in Eq. (46), one may calculate the electric charge carried by each quasiparticle:

$$
\begin{aligned}
& Q_{e}=\left(q_{2}, q_{1}\right)\left(K_{\partial}\right)^{-1}(1,0)^{T}=\frac{q_{1}}{2}, \\
& Q_{m}=\left(q_{2}, q_{1}\right)\left(K_{\partial}\right)^{-1}(0,1)^{T}=\frac{q_{2}}{2} .
\end{aligned}
$$

Physically, both $e$ and $m$ quasiparticles can always attach trivial identity particles to change their charges by an arbitrary integer so that $q_{1}$ and $q_{2}$ are integers $\bmod 2$; namely,

$$
q_{1} \sim q_{1}+2, \quad q_{2} \sim q_{2}+2 .
$$

For this reason, it is sufficient to merely consider the following four choices: $\left(q_{2}, q_{1}\right)=(0,0),(0,1),(1,0),(1,1)$. The first three choices can be realized on a $2 \mathrm{D}$ plane without breaking time-reversal symmetry since the Hall conductance $\quad \sigma_{x y}=(1 / 2 \pi) q^{T}\left(K_{\partial}\right)^{-1} q=\left(q_{1} q_{2} / 2 \pi\right)=0$ 
on a $2 \mathrm{D}$ plane. However $(1,1)$ necessarily breaks timereversal symmetry on a 2D plane since its Hall conductance $\sigma_{x y}=(1 / 2 \pi) q^{T}\left(K_{\partial}\right)^{-1} q=(1 / 2 \pi) q_{1} q_{2}=1 / 2 \pi$ is nonzero. Thus, there is a nonvanishing chiral charge flow on the $1 \mathrm{D}$ edge of the 2D plane. However, it does not break time-reversal symmetry on the surface since chiral charge flow is not a physical observable on the surface. Therefore, the charge assignment $(1,1)$ faces obstruction in being realized on a 2D plane with $\mathrm{U}(1) \rtimes Z_{2}^{T}$ symmetry and this obstruction leads to a BTI in which both $e$ and $m$ carry halfcharge on the surface. Note that the $q_{1}$-coupling terms in Eqs. (47) and (48) change sign under time-reversal transformation. However, the sign can be removed through shifting $q_{1}$ to $-q_{1}$ following the identification in Eq. (49).

In a real transport experiment, one may explicitly break time-reversal symmetry along the normal direction on the surface with charge assignment $(1,1)$ and put the $3 \mathrm{D}$ system in a slab geometry. The surface quantum Hall conductance is quantized at $1 / 2 \pi$, which corresponds to a surface response action in the form of a Chern-Simons term: $(1 / 4 \pi) A_{\mu} \partial_{\nu} A_{\lambda} \epsilon^{\mu \nu \lambda}$. It can be formally extended to a bulk $\Theta$ term, i.e., Eq. (41) with $\Theta$ angle given by $\Theta=2 \pi \bmod 4 \pi$ and the following generic relation:

$$
\Theta=4 \pi^{2} \sigma_{x y} .
$$

The $4 \pi$ periodicity corresponds to even-quantized Hall conductance $\sigma_{x y}=(1 / 2 \pi) \times 2 k$, which can be realized in purely $2 \mathrm{D}$ bosonic systems. A projective construction on such a BTI with $\Theta$ response has been made in detail in Ref. [49].

Physically, $e$ and $m$ particles can be regarded as ends of condensed vortex lines here, and we may think these invisible vortex lines carry integer charge and form a nontrivial 1D BTI phase. Thus, it is also not a surprise that the end of these vortex lines will carry half-charge.

\section{Many-to-one correspondence between surface and bulk}

The above discussion illustrates how the surface $Z_{2}$ topological order arise with unusual U(1) symmetry transformations. As a matter of fact, the surface may be of different kinds while all share the same bulk that only supports trivial boson excitations. In other words, the surface topological order may be much richer.

One may replace the $K$ matrix in Eq. (42) by

$$
K=\left(\begin{array}{ll}
0 & 1 \\
1 & p
\end{array}\right),
$$

where $p$ is a nonzero positive integer. The surface term Eq. (46) now becomes

$$
\mathcal{L}_{\partial}=i \frac{p}{2 \pi} \epsilon^{\mu \nu \lambda} \tilde{a}_{\mu}^{2} \partial_{\nu} a_{\lambda}^{2}
$$

which corresponds to a $Z_{p}$ topological order on the surface labeled by $K_{\partial}=\left(\begin{array}{ll}0 & p \\ p & 0\end{array}\right)$.

There are $p^{2}$ types of quasiparticles (including the trivial particle), which are labeled by quasiparticle vector $l=\left(l_{1}, l_{2}\right)^{T}$, with $l_{1}, l_{2}=0,1, \ldots, p-1$. The $l$ th quasiparticle carries the electric charge

$$
Q_{l}=q^{T} K_{\partial}^{-1} l=\frac{1}{p}\left(q_{1} l_{2}+q_{2} l_{1}\right) .
$$

Physically, all quasiparticles can attach trivial identity particles such that their charges can be changed by any integer. Therefore, the following identification conditions exist:

$$
q_{1} \sim q_{1}+p, \quad q_{2} \sim q_{2}+p .
$$

We note that $q_{1}$-coupling terms in Eqs. (47) and (48) change sign under time-reversal transformation. However, the sign can be removed through shifting $q_{1}$ to $-q_{1}$ following the identification in Eq. (54). Therefore, bulk time-reversal symmetry requires that $p=$ even and only two choices for the integer $q_{1}$ are legitimate: $q_{1}=0 \bmod p$, $\frac{p}{2} \bmod p$.

To determine whether or not the bulk is trivial, one may again examine the surface Hall conductance which is quantized at odd for nontrivial bulk. All even-quantized parts can be removed by stacking several U(1) SPT states on the surface. At present, the Hall conductance is given by $\sigma_{x y}=(1 / 2 \pi) q^{T} K_{\partial}^{-1} q=(1 / 2 \pi)\left(2 q_{1} q_{2} / p\right)$. Combined with the relation in Eq. (50), we end up with Table II. As a side note, in the first two cases, by means of the identification in Eq. (54), $q_{2}$ may be shifted by $p$. Since $p$ is even in these two cases, the even or odd property of $q_{2}$ is unchanged. Thus, these two cases are consistent with the identification conditions.

When $p=2, q_{1}=q_{2}=1$, the theory goes back to Sec. VIB. It is clear that the surface $Z_{2}$ topological order obtained in Sec. VIB is just one possible surface of BTI states, which manifests the physics of many-to-one correspondence between surface and bulk. For example, we may choose $p=4, q_{1}=2, q_{2}=1$ such that the bulk is a BTI

TABLE II. The surface of the first BTI root states (shown in the first row) labeled by three integers $\left(p, q_{1}, q_{2}\right)$.

\begin{tabular}{lcc}
\hline \hline$p$ & $q_{1}, q_{2}$ & Bulk \\
\hline$p=$ even & $q_{1}=\frac{p}{2} \bmod p, q_{2}=$ odd & The first BTI root states \\
$p=$ even & $q_{1}=\frac{p}{2} \bmod p, q_{2}=$ even & Trivial states \\
$p=$ even & $q_{1}=0 \bmod p$, & Trivial states \\
& $q_{2}=$ any integers & \\
$p=$ even & $q_{1} \neq 0 \bmod p, q_{1} \neq \frac{p}{2} \bmod p$, & $Z_{2}^{T}$ is broken \\
& $q_{2}=$ any integers & \\
$p=$ odd & $q_{1}, q_{2}=$ any integers & $Z_{2}^{T}$ is broken \\
\hline \hline
\end{tabular}


state with nontrivial Witten effect. The electric charge carried by totally $4^{2}-1=15$ nontrivial quasiparticles can be calculated by Eq. (53): $Q_{l}=\left(l_{2} / 2\right)+\left(l_{1} / 4\right)$. Such an assignment of fractional charge on quasiparticles of $Z_{4}$ topological order cannot be realized on a $2 \mathrm{D}$ plane unless breaking time-reversal symmetry.

\section{BOSONIC TOPOLOGICAL INSULATORS FROM PURE $b \wedge d a$ TERM (II)}

In Sec. VI, we show that the first BTI root state can be obtained by a pure $b \wedge d a$ topological term where $\mathrm{U}(1)$ charge symmetry is defined in an unusual way. In the following, we continue to show that the second BTI root state (the second row in Table I) can be obtained by a pure $b \wedge d a$ topological term where time-reversal symmetry is defined in an unusual way.

\section{A. $\mathbb{Z}_{2}$ nature of bulk time-reversal symmetry definition}

Let us consider the $K$ matrix in the form of Eq. (18). A time-reversal transformation acting on gauge fields $a_{\mu}^{I}, b_{\mu \nu}^{I}$ can be formally expressed as

$$
\begin{array}{r}
\mathcal{T} a_{0}^{I} \mathcal{T}^{-1}=T_{I J}^{a} a_{0}^{J}, \quad \mathcal{T} b_{0 i}^{I} \mathcal{T}^{-1}=T_{I J}^{b} b_{0 i}^{J}, \\
\mathcal{T} a_{i}^{I} \mathcal{T}^{-1}=-T_{I J}^{a} a_{i}^{J},
\end{array}
$$

where $T^{a}$ and $T^{b}$ are two integer-valued matrices. In the following, we simply call $T^{a}$ and $T^{b}$ " $T$ matrices." After transforming twice, all gauge variables are unchanged, so we have the constraint $\left(T^{a}\right)^{2}=\left(T^{b}\right)^{2}=1$. It indicates that $\left|\operatorname{det} T^{a}\right|=\left|\operatorname{det} T^{b}\right|=1$, and both matrices belong to a subset of $\mathbb{G} \mathbb{L}(N, \mathbb{Z})$ group.

After $\mathbb{G L}$ transformations and time-reversal transformation, the $K$ matrix is transformed to a new one, but $|\operatorname{det} K|=1$ is still valid such that the bulk still merely supports trivial gapped boson excitations as before. From this perspective, the bulk always keeps time-reversal symmetry although the formal expression of the Lagrangian is given by a new $K$ matrix.

On the other hand, one may apply arbitrary $\mathbb{G} \mathbb{L}(N, \mathbb{Z})$ transformations on both sides of all equations in Eqs. (55) and (56). Using the notation in Eq. (21), we obtain the following equations:

$$
\begin{gathered}
\mathcal{T} \underline{a}_{0}^{I} \mathcal{T}^{-1}=\underline{T}_{I J}^{a} \underline{a}_{0}^{J}, \quad \\
\mathcal{T} \underline{b}_{0 i}^{I} \mathcal{T}^{-1}=\underline{T}_{I J}^{b} \underline{b}_{0 i}^{J}, \\
\mathcal{T} \underline{a}_{i}^{I} \mathcal{T}^{-1}=-\underline{T}_{I J}^{a} \underline{a}_{i}^{J}, \quad \mathcal{T} \underline{b}_{i j}^{I} \mathcal{T}^{-1}=-\underline{T}_{I J}^{b} \underline{b}_{i j}^{J},
\end{gathered}
$$

where two $T$ matrices are transformed to two new ones:

$$
\underline{T}^{a} \stackrel{\underline{\operatorname{def}}}{=} M^{-1} T^{a} M, \quad \underline{T^{b}} \underline{\underline{\operatorname{def}}} W^{-1} T^{b} W .
$$

By keeping $|\operatorname{det} W|=|\operatorname{det} M|=1$ in mind, it is clear that the \pm sign of the determinant of the $T$ matrices is manifestly invariant under an arbitrary sequence of formal
$\mathbb{G L}$ transformations. For the sake of convenience, we introduce a notation $(a, b)$ that denotes such \pm signs of determinants:

$$
(a, b) \underline{\underline{\operatorname{def}}}\left(\operatorname{sign} \text { of } \operatorname{det} T^{a}, \text { sign of } \operatorname{det} T^{b}\right) .
$$

Because of the presence of this invariant, we are able to understand the usual time-reversal transformation defined in Eqs. (28) and (29) in a much more general background. In terms of $T$ matrices, the usual time-reversal transformation defined in Eqs. (28) and (29) is denoted by

$$
T^{a}=-T^{b}=\operatorname{diag}(1,1, \ldots, 1)_{N \times N}
$$

where the $K$ matrix is fixed as a unit matrix shown in Eq. (18). This specific form of usual time-reversal transformation in a given basis (i.e., $K=\rrbracket$ ) can be generalized and replaced by the following invariant:

$$
(a, b)=\left(1,(-1)^{N}\right),
$$

which is a universal property of all specific forms of usual time-reversal transformations.

To summarize, we consider a 3D bulk state described by an $N$-component $b \wedge d a$ term labeled by $K$ with $|\operatorname{det} K|=1$. There are $T^{a}$ and $T^{b}$ matrices that define time-reversal transformations. As discussed before, for the purpose of exploring nontrivial SPT states, we consider the cases that have a symmetry-preserving surface. Then, if $(a, b)=\left(1,(-1)^{N}\right)$, the state admits a usual time-reversal transformation and thereby a trivial SPT state. If $(a, b) \neq\left(1,(-1)^{N}\right)$, the state is a nontrivial SPT state. From this point of view, a $\mathbb{Z}_{2}$ classification is obtained by attempting to change the definition of time-reversal transformations. Along this line of thinking, in Sec. VII B, we will $N=2$ as a simple example that reproduces the BTI state labeled by the first $\mathbb{Z}_{2}$ index introduced in Sec. I.

\section{B. Example with $N=\mathbf{2}$}

We still start with a $b \wedge d a$ theory with the $K$ matrix given by Eq. (42). The total Lagrangian is given by Eq. (43). However, at present, the time-reversal transformation is defined as

$$
\begin{array}{cc}
\mathcal{T} a_{0}^{1} \mathcal{T}^{-1}=a_{0}^{1}, & \mathcal{T} a_{i}^{1} \mathcal{T}^{-1}=-a_{i}^{1}, \\
\mathcal{T} b_{0, i}^{1} \mathcal{T}^{-1}=b_{0, i}^{1}, & \mathcal{T} b_{i, j}^{1} \mathcal{T}^{-1}=-\underline{b}_{i, j}^{1}, \\
\mathcal{T} a_{0}^{2} \mathcal{T}^{-1}=-a_{0}^{2}, & \mathcal{T} a_{i}^{2} \mathcal{T}^{-1}=a_{i}^{2}, \\
\mathcal{T} b_{0, i}^{2} \mathcal{T}^{-1}=-b_{0, i}^{2}, & \mathcal{T} b_{i, j}^{2} \mathcal{T}^{-1}=b_{i, j}^{2},
\end{array}
$$

which is different from the usual definition Eqs. (28) and (29). The associated $T$ matrices of the time-reversal operator $\mathcal{T}$ are given by 


$$
T^{a}=\left(\begin{array}{cc}
1 & 0 \\
0 & -1
\end{array}\right), \quad T^{b}=\left(\begin{array}{cc}
1 & 0 \\
0 & -1
\end{array}\right) .
$$

This definition of time-reversal symmetry transformation is labeled by $(a, b)=(-1,-1)$, which is nontrivial according to Eq. (61). Under the time-reversal transformation, the $b \wedge d a$ term labeled by $K$ is transformed to the term labeled by $K^{\prime}=\left(\begin{array}{cc}0 & 0 \\ 1 & -2\end{array}\right)$. At first glance, time-reversal symmetry is broken in the bulk. However, two $b \wedge d a$ theories labeled by $K^{\prime}$ and $K$ are $\mathbb{G L}$ equivalent by using

$$
W=\left(\begin{array}{cc}
1 & 0 \\
0 & -1
\end{array}\right), \quad M=\left(\begin{array}{cc}
-1 & 0 \\
0 & 1
\end{array}\right)
$$

defined in Eq. (22). More explicitly, these $\mathbb{G} \mathbb{L}$ transformations lead to a sign change in both $b_{\mu \nu}^{2}$ and $a_{\mu}^{1}$ while $b_{\mu \nu}^{1}$ and $a_{\mu}^{2}$ are invariant:

$$
\begin{aligned}
b_{\mu \nu}^{2} & \rightarrow-b_{\mu \nu}^{2}, & a_{\mu}^{1} & \rightarrow-a_{\mu}^{1}, \\
b_{\mu \nu}^{1} & \rightarrow b_{\mu \nu}^{1}, & a_{\mu}^{2} & \rightarrow a_{\mu}^{2} .
\end{aligned}
$$

Let us move on to the surface theory Eq. (46). According to the transformation of $b_{\mu \nu}^{2}$ in Eq. (65), the time-reversal transformation rule of $\tilde{a}_{\mu}^{2}$ defined in Eq. (45) is automatically fixed:

$$
\mathcal{T} \tilde{a}_{0}^{2} \mathcal{T}^{-1}=-\tilde{a}_{0}^{2}, \quad \mathcal{T} \tilde{a}_{i}^{2} \mathcal{T}^{-1}=\tilde{a}_{i}^{2}
$$

Because of the time-reversal transformations in Eqs. (69) and (64), both $m$ and $e$, which carry unit gauge charges of $\tilde{a}_{\mu}^{2}$ and $a_{\mu}^{2}$, respectively, are pseudolike particles on the surface. Under these time-reversal transformations, a minus sign appears in the Chern-Simons term in Eq. (46). Despite that, the surface state does not break time reversal. More precisely, the appearance of this minus sign leaves the set of surface physical observables in Definition 1 unaffected by noting that there is a $\mathbb{G} \mathbb{L}$ equivalence relation: $P^{T}\left(-K_{\partial}\right) P=K_{\partial}$, where $P=\left(\begin{array}{cc}1 & 0 \\ 0 & -1\end{array}\right)$. Therefore, the surface is symmetric under time-reversal transformation.

It is also beneficial to investigate the equations of motion (EOMs) of gauge fields under time-reversal symmetry. By adding two quasiparticle currents, $j_{\mu}^{e} a_{\mu}^{2}+j_{\mu}^{m} \tilde{a}_{\mu}^{2}$, where $j_{\mu}^{e}$ and $j_{\mu}^{m}$ are $e$-particle and $m$-particle currents, respectively, the EOMs of gauge fields are given by (only the zero component is shown here without loss of generality) $\pi \rho^{e}=\nabla \times \tilde{\mathbf{a}}^{2} \underline{\underline{\operatorname{def}}} \tilde{\mathcal{B}}, \pi \rho^{m}=\nabla \times \mathbf{a}^{2} \underline{\underline{\operatorname{def}} \mathcal{B},}$ where $\rho^{e}=j_{0}^{e}$ and $\rho^{m}=j_{0}^{m}$ are density variables of $e$ and $m$, respectively. $\tilde{\mathcal{B}}$ and $\mathcal{B}$ are the magnetic flux strength of $\tilde{\mathbf{a}}^{2}$ and $\mathbf{a}^{2}$, respectively. Under time reversal, $\rho_{e}$ and $\rho_{m}$ change sign since they are pseudolike: $\rho_{e} \rightarrow-\rho_{e}, \rho_{m} \rightarrow-\rho_{m}$. On the other hand, both $\tilde{\mathcal{B}}$ and $\mathcal{B}$ are unchanged under time-reversal transformations Eqs. (69) and (64). It seems that EOMs break time reversal. However, time-reversal symmetry is still unbroken due to the very existence of the 3D bulk. More concretely, the 3D bulk can source a trivial particle that carries two units of $a_{\mu}^{2}$ gauge charge. One may attach this trivial particle to a time-reversal partner of $e$ particles on the surface, rendering $-\rho_{e}+2 \rho_{e}=\rho_{e}$. Likewise, the 3D bulk can source a trivial particle that carries two units of $\tilde{a}_{\mu}^{2}$ gauge charge. One may attach this trivial particle to a time-reversal partner of $m$ particles, rendering $-\rho_{m}+2 \rho_{m}=\rho_{m}$. As a result, both EOMs respect time-reversal symmetry.

However, this $Z_{2}$ topological order state on a 2D plane (i.e., no 3D bulk) necessarily breaks time-reversal symmetry. In the absence of 3D bulk, all trivial particles that are used to change sign of $\rho_{e}$ and $\rho_{m}$ can only come from the $2 \mathrm{D}$ state itself. Consequently, the magnetic fluxes generated by these trivial particles will change $\int d x d y \tilde{\mathcal{B}}$ and $\int d x d y \mathcal{B}$ to $\int d x d y \tilde{\mathcal{B}}+2 \pi$ and $\int d x d y \mathcal{B}+2 \pi$, respectively. Thus, EOMs always break time-reversal symmetry.

In summary, following the definition of obstruction in Definition 2, the 3D bulk is a nontrivial SPT state, i.e., a BTI state. Physically, the unique way to realize such a time-reversal symmetry on gauge fields, i.e., Eqs. (69) and (64), is to consider that both $e$ and $m$ are Kramers doublets, and $\rho_{e}, \rho_{m}$ should be regarded as spin density $S_{z}$ of a spin- $1 / 2$ particle. We note that by "spin $1 / 2$ " here, we really mean a projective representation of time-reversal symmetry and it has nothing to do with spin-rotational symmetry. In the bulk, all particles must carry a linear representation of time-reversal symmetry; therefore, the spin-1/2 particle on the surface cannot be screened. Other possibilities of Kramers degeneracy assignment (e.g., $e$ is a Kramers doublet while $m$ is a Kramers singlet) can be realized on a 2D plane without breaking time-reversal symmetry $[48,97]$. This obstruction provides us a physical way to understand the nontrivial BTI root phase generated by exotic time-reversal symmetry. Indeed, both $e$ and $m$ particles can be regarded as ends of vortex lines that are condensed and invisible in the bulk. In terms of a simple physical picture, we may think these invisible vortex lines carry integer spin and form a nontrivial 1D SPT phase, e.g., the Haldane phase. Therefore, it is not a surprise that the ends of these vortex lines carry half-integer spins which form Kramers doublets under time-reversal symmetry.

\section{VIII. $Z_{N}$ SPT IN THREE DIMENSIONS: BEYOND GROUP COHOMOLOGY THEORY}

In this section, we use the one component action Eq. (14) to discuss possible $Z_{N}$ symmetry-protected phases beyond the group cohomology class. Let us that assume a generic $Z_{N} \mathrm{SPT}$ in 3D can be described by 


$$
\mathcal{L}=i \frac{1}{4 \pi} a_{\mu} \partial_{\nu} b_{\lambda \rho} \epsilon^{\mu \nu \lambda \rho}+i \frac{\Lambda}{16 \pi} b_{\mu \nu} b_{\lambda \rho} \epsilon^{\mu \nu \lambda \rho},
$$

where only one component is taken into account for convenience. Here, $a_{\mu}$ and $b_{\mu \nu}$ are still noncompact and compact, respectively. In contrast to the previous discussion of $\mathrm{U}(1) \rtimes Z_{2}^{T}$, where nonvanishing quantized $\Lambda$ needs the help of time-reversal symmetry, $\Lambda$ in $Z_{N}$ SPT is supposed to be quantized even without the help of timereversal symmetry. Following Ref. [34], we consider the following gauge coupling to "probe the $Z_{N}$ SPT order":

$$
\mathcal{L}_{\text {coupling }}=i \frac{1}{4 \pi} B_{\mu \nu} \partial_{\lambda} a_{\rho} \epsilon^{\mu \nu \lambda \rho}+i \frac{N}{4 \pi} B_{\mu \nu} \partial_{\lambda} A_{\rho} \epsilon^{\mu \nu \lambda \rho} .
$$

Several explanations are in order. First, the $B_{\mu \nu}$ gauge field in the $B \wedge d a$ term is a "2-form compact probe field" that minimally couples to strings ( $2 \pi$ vortex lines in the ground state). It is this type of coupling that is missed in the Dijkgraaf-Witten gauge theory [98] since $H^{4}\left[Z_{N}, \mathrm{U}(1)\right]=\mathbb{Z}_{1}$. The term $B \wedge d A$ can be viewed as a Higgs condensate term [34], where $A_{\mu}$ is a 1-form compact gauge field introduced by Hubbard-Stratonovich transformation. By means of this term, the probe field $B_{\mu \nu}$ is naturally Higgsed to a $Z_{N}$ discrete gauge field.

Now, we are in a position to integrate out all SPT degrees of freedom. Integrating the noncompact field $a_{\mu}$ renders $b_{\mu \nu}=B_{\mu \nu}$. Consequently, we end up with an action in the background fields $B$ and $A$ [71]:

$$
\begin{aligned}
S & =i \int d^{4} x \frac{N}{4 \pi} B_{\mu \nu} \partial_{\lambda} A_{\rho} \epsilon^{\mu \nu \lambda \rho}+i \int d^{4} x \frac{\Lambda}{16 \pi} B_{\mu \nu} B_{\lambda \rho} \epsilon^{\mu \nu \lambda \rho} \\
& =i \frac{N}{2 \pi} \int B \wedge d A+i \frac{\Lambda}{4 \pi} \int B \wedge B
\end{aligned}
$$

All possible values of $\Lambda$ can be found by using the procedures in Sec. IV B. Finally, we end up with the following quantization condition (more details are found in Appendix B):

$$
\Lambda / N \in \mathbb{Z}
$$

The constraints Eqs. (B2) and (71) suggest $Z_{N}$ different SPT states protected by $Z_{N}$ symmetries in three dimensions.

However, since the probe field here is a 2-form gauge field with a flat connection, it cannot be regarded as the symmetry twist of a usual condensed matter system where symmetry charges are carried by pointlike particles. Instead, we need to consider a system consisting of stringlike objects carrying global symmetry quantum numbers, for example, in the context of "generalized global symmetries" in string theory reported recently by Davide et al. [99]. Although for solid-state systems we are not aware of how to prepare stringlike objets carrying global quantum numbers, we hope that certain artificial quantum systems, e.g., cold atom systems, might be able to realize these exotic quantum phases.

\section{CONCLUSIONS}

In this paper, based on a physical process called "vortexline condensation" in three-dimensional superfluids, we construct a bulk dynamical TQFT description Eq. (1) of all three bosonic topological insulator states (BTI). The schematic phase diagram is shown in Fig. 1. Such a physical way of thinking allows us to understand the physical meaning of each gauge field variable, and, most importantly, symmetry definitions in the bulk. Our method sheds light on a more challenging question of how to design microscopic interactions to realize BTI states in solid-state materials or ultracold-atom experiments. In particular, it is quite interesting to explore the possible interaction terms to realize the linking Berry phase contributed by $b \wedge b$ as shown in Fig. 2. For those two BTI states (Secs. VI and VII) that do not need a $b \wedge b$ term, one may attach either charge or spin degrees of freedom to vortex lines, which may result in BTI states after vortex lines condense.

We show that one of the three BTI states requires a nontrivial existence of a $b \wedge b$ term, which is beyond group cohomology theory. The remaining two states are within group cohomology theory and can be constructed from the pure $b \wedge d a$ term. In contrast to previous works of BTI where the surface topological order is always $Z_{2}$ topological order, now the surface topological order of the BTI state within group cohomology classification can be a generic $Z_{p}$ topological order with even $p$, as shown in Sec. VI. This many-to-one correspondence between surface and bulk explicitly indicates that knowledge about bulk field theory is highly desirable, which is also explained in detail in Sec. I. In addition to BTI, applying our construction of BTI to 3D SPT with $Z_{N}$ unitary symmetry suggests some nontrivial $Z_{N}$ SPT state in three dimensions beyond the group cohomology classification. However, in contrast to the usual quantum systems where the global symmetry quantum number is carried by pointlike particles, the new class of $Z_{N}$ SPT phases proposed here requires a stringlike object to carry the global symmetry quantum number. Finally, based on the results we obtain, we conjecture that all SPT phases described by Eq. (1) with a nontrivial $b \wedge b$ term are generally beyond the group cohomology classification.

In the future, it will be interesting to apply such a physical derivation of bulk dynamical TQFT to other SPT states, even including fermionic SPT states where a spin manifold is required. A challenging problem is the bulk dynamical TQFT (not response theory) description of FTI both in free-fermionic [6-12] and interacting cases [100-103]. There have been many previous important efforts, such as Refs. [104,105]. In Ref. [106], functional bosonization techniques are applied and the $b \wedge b$ term 
appears. We believe that the basic methodology we present in our work combined with the previous efforts will shed light on this hard problem.

\section{ACKNOWLEDGMENTS}

We thank Meng Cheng, Chao-Ming Jian, Joseph Maciejko, Xiao-Gang Wen, and Shing-Tung Yau for enlightening discussion and/or comments. We thank Ching Hua Lee and Gang Chen for many valuable suggestions on the manuscript. We also thank Princeton Center for Theoretical Science at Princeton University for hospitality during the "Symmetry in Topological Phases" Workshop, where this work was initiated. P. Y. especially acknowledges Professor Zheng-Yu Weng at Institute for Advanced Study in Tsinghua University in Beijing where the work was done in part. Research at Perimeter Institute is supported by the Government of Canada through Industry Canada and by the Province of Ontario through the Ministry of Economic Development and Innovation.

\section{APPENDIX A: QUANTIZATION CONDITIONS AND EVALUATIONS OF PARTITION FUNCTION ON CLOSED MANIFOLDS}

In Sec. IV B, we obtain the quantization condition Eq. (24) based on the physical approach, namely, the microscopic origin vortex-line condensation. Although in the whole derivation of the third BTI root state (see Table I) we consider this physical approach and $a$ is noncompact, in this Appendix, we derive the same quantization condition in a mathematical setting that is independent of the microscopic origin. First of all, we assume that both $b$ and $a$ satisfy the following compactification conditions:

$$
\begin{gathered}
\iiint_{\mathcal{V}^{\prime}} d b=2 \pi \times \text { integer, } \\
\oiint_{\mathcal{S}} d a=2 \pi \times \text { integer, }
\end{gathered}
$$

where, both the 3D manifold $\mathcal{V}^{\prime}$ and the 2D manifold $\mathcal{S}$ are closed. Let us consider the partition function

$$
\mathbf{Z}=\int \mathcal{D}[a] \mathcal{D}[b] e^{-S},
$$

where the classical action $S$ is given by Eq. (23). Integration over $d a$ is a Poisson summation, which directly leads to the quantization condition on $b$ given by Eq. (24). Therefore, both ways give the same answer. The treatment in the main text can be viewed as a semiclassical way, while the new way is more rigorous since the nontrivial shift in Eq. (16) generally renders compactification of $a$ once $b$ is assumed to be compact. A similar line of thinking was given recently in Ref. [107].
We also check whether the addition of the $b \wedge b$ term induces fractionalization, namely, topological order in the bulk. Following the spirit of the Laughlin thought experiment for probing electron fractionalization in fractional quantum Hall effects, we apply an external gauge field that minimally couples to the conserved currents of matter fields, i.e., bosonic point particles and strings (vortex lines). In the present $3 \mathrm{D}$ state, we add a 2 -form external gauge field $B$ through the minimal coupling term $(1 / 2 \pi) B \wedge d a$, where $(1 / 2 \pi)^{*} d a$ denotes the 2 -form string (vortex-line) current. The asterisk $\left({ }^{*}\right)$ denotes Hodge dual. We choose $B$ instead of the usual 1-form external electromagnetic field $A$ to probe fractionalization because in the $3 \mathrm{D}$ state anyons do not exist while possible fractionalization comes only from fractionalization of flux strength of vortex lines; namely, $2 \pi$ becomes $2 \pi / k$, with $k>1, k \in \mathbb{Z}$.

Then the ground-state degeneracy (GSD) ratio in a given space-time manifold between the theories with and without $b \wedge b$ is given by the ratio of partition functions in the presence of external gauge field $B$ :

$$
\frac{\mathrm{Z}}{\mathrm{Z}_{0}}=\frac{1}{\mathrm{Z}_{0}} \int \mathcal{D}[a] \mathcal{D}[b] e^{-i \int[(1 / 2 \pi) b \wedge d a+(\Lambda / 4 \pi) b \wedge b-(1 / 2 \pi) a \wedge d B]},
$$

where $Z_{0}$ is given by $Z_{0}=Z[\Lambda=0]$ by definition. Integration over $a$ is a Poisson summation, which leads to

$$
\oiint_{\mathcal{S}}(b+B)=2 \pi \times \text { integer }
$$

where $\mathcal{S}$ is a closed 2D manifold forming a surface of a 3D space $\mathcal{V}$, i.e., $\mathcal{S}=\partial \mathcal{V}$.

We note that the probe field $B$ satisfies the quantization condition:

$$
\oiint_{\mathcal{S}} B=2 \pi \times \text { integer. }
$$

Thus, we set $B=b$ up to gauge transformation (including the large one) in the $b \wedge b$ term, which leads to the following result:

$$
\frac{\mathrm{Z}}{\mathrm{Z}_{0}}=e^{i \int(\Lambda / 4 \pi) B \wedge B}
$$

In space-time with different topology, we may calculate the ratio that is always a phase factor with unit length, i.e., $\left|Z / Z_{0}\right|=1$. For example, one may choose $\mathbb{T}_{0 x} \times \mathbb{T}_{y z}$ topology and calculate the ratio. The calculation is completely the same as in Appendix B 1 by just replacing $b$ by $B$. In summary, the GSD ratio in the presence of $b \wedge b$ is still one in all kinds of space-time topology, since the pure $b \wedge d a$ theory is at level 1 with GSD $=1$. Thus, the 
addition of $b \wedge b$ does not induce new GSD and thus fractionalization in our theory.

\section{APPENDIX B: SOME TECHNICAL DETAILS}

\section{Physical understanding of Eq. (25)}

We consider the two tori $\mathbb{T}_{0 x}$ and $\mathbb{T}_{y z}$. The former is formed by imaginary time direction and $x$ direction. The latter is formed by $y$ and $z$ directions. In both tori, we have the following constraints due to the condition Eq. (24):

$$
\begin{aligned}
& \iint_{\mathbb{T}_{0 x}} b_{0 x} d \tau d x=2 \pi \times \mathcal{N}_{0 x}, \\
& \iint_{\mathbb{T}_{y z}} b_{y z} d y d z=2 \pi \times \mathcal{N}_{y z},
\end{aligned}
$$

where $\tau$ is imaginary time. Note that the above two integrals are performed at fixed $y, z$ and fixed $\tau, x$, respectively. Since the integrals in the lhs of both equations are smooth functions of space-time, the integers $\mathcal{N}_{0 x}\left(\mathcal{N}_{y z}\right)$ should be independent of the coordinates $y$ and $z$ ( $\tau$ and $x$ ).

Then, one may reformulate the $b \wedge b$ term as

$$
\begin{aligned}
i \frac{\Lambda}{16 \pi} \int d^{4} x \epsilon^{\mu \nu \lambda \rho} b_{\mu \nu} b_{\lambda \rho}= & i \frac{\Lambda}{16 \pi} \iint_{\mathbb{T}_{0 x}} d \tau d x b_{0 x} \iint_{\mathbb{T}_{y z}} d y d z b_{y z} \epsilon^{0 x y z}+(x 0 y z) \\
& +(0 x z y)+(x 0 z y)+(y z 0 x)+(y z x 0)+(z y 0 x)+(z y x 0) \\
= & i \frac{8 \Lambda}{16 \pi} \iint_{\mathbb{T}_{0 x}} d \tau d x b_{0 x} \iint_{\mathbb{T}_{y z}} d y d z b_{y z} \\
= & i \frac{\Lambda}{2 \pi} 2 \pi \mathcal{N}_{0 x} 2 \pi \mathcal{N}_{y z} \\
= & i 2 \pi \Lambda \mathcal{N}_{0 x} \mathcal{N}_{y z} .
\end{aligned}
$$

By noting that the partition function is invariant if $\int \mathcal{L}$ is shifted by $2 \pi$ in a quantized theory, we end up with the periodicity shift shown in Eq. (25).

\section{Derivation of Eq. (71)}

The condition Eq. (24) leads to $\mathcal{N}_{0 x}$ and $\mathcal{N}_{y z}$ (see Appendix B 1) quantized at $1 / N$ such that the periodicity of $\Lambda$ is

$$
\Lambda \rightarrow \Lambda+N^{2}
$$

On the other hand, as a discrete gauge theory, the $B \wedge B$ term should also be invariant up to $2 \pi$ under large gauge transformation: $B_{\mu \nu} \rightarrow B_{\mu \nu}+\delta B_{\mu \nu}$, where $\delta B_{\mu \nu}$ satisfies $\int_{\mathcal{S}} \delta B_{\mu \nu} \mathcal{S}^{\mu \nu}=2 \pi \times$ integer (there is no implicit summation over indices $\mu, \nu$ here). Without loss of generality, we still consider the pair of tori $\mathbb{T}_{0 x}$ and $\mathbb{T}_{y z}: \int_{\mathbb{T}_{0 x}} \delta B_{0 x} d \tau d x=$ $2 \pi \times \tilde{\mathcal{N}}_{0 x}, \int_{\mathbb{T}_{y z}} \delta B_{y z} d y d z=2 \pi \times \tilde{\mathcal{N}}_{y z}, \quad$ The additional terms $\delta S$ arising from the large gauge transformation are collected as follows:

$$
\begin{aligned}
S+\delta S= & i \frac{\Lambda}{16 \pi} \iint_{\mathbb{\mathbb { T }}_{0 x}} d \tau d x\left(B_{0 x}+\delta B_{0 x}\right) \iint_{\mathbb{T}_{y z}} d y d z\left(B_{y z}+\delta B_{y z}\right) \epsilon^{0 x y z} \\
& +(x 0 y z)+(0 x z y)+(x 0 z y)+(y z 0 x)+(y z x 0)+(z y 0 x)+(z y x 0) \\
= & S+\frac{8 \Lambda}{16 \pi}\left(\frac{2 \pi}{N} \mathcal{N}_{0 x} 2 \pi \tilde{\mathcal{N}}_{y z}+2 \pi \tilde{\mathcal{N}}_{0 x} \frac{2 \pi}{N} \mathcal{N}_{y z}+2 \pi \tilde{\mathcal{N}}_{0 x} 2 \pi \tilde{\mathcal{N}}_{y z}\right) \\
= & S+2 \pi \Lambda\left(\frac{1}{N} \mathcal{N}_{0 x} \tilde{\mathcal{N}}_{y z}+\frac{1}{N} \tilde{\mathcal{N}}_{0 x} \mathcal{N}_{y z}+\tilde{\mathcal{N}}_{0 x} \tilde{\mathcal{N}}_{y z}\right)
\end{aligned}
$$

To keep the quantum theory invariant under the large gauge transformation, $\delta S$ must equal to integer $\times 2 \pi$, leading to the quantization condition Eq. (71). 
[1] Z.-C. Gu and X.-G. Wen, Tensor-entanglement-filtering renormalization approach and symmetry-protected topological order, Phys. Rev. B 80, 155131 (2009).

[2] X. Chen, Z.-C. Gu, Z.-X. Liu, and X.-G. Wen, Symmetry protected topological orders and the group cohomology of their symmetry group, Phys. Rev. B 87, 155114 (2013).

[3] X. Chen, Z.-C. Gu, and X.-G. Wen, Local unitary transformation, long-range quantum entanglement, wave function renormalization, and topological order, Phys. Rev. B 82, 155138 (2010).

[4] X. Chen, Z.-C. Gu, Z.-X. Liu, and X.-G. Wen, Symmetryprotected topological orders in interacting bosonic systems, Science 338, 1604 (2012); X. L. Qi, Symmetry meets topology, Science 338, 1550 (2012).

[5] T. Senthil, Symmetry protected topological phases of quantum matter, arXiv:1405.4015.

[6] J.E. Moore and L. Balents, Topological invariants of time-reversal-invariant band structures, Phys. Rev. B 75, 121306 (2007).

[7] L. Fu, C. L. Kane, and E. J. Mele, Topological Insulators in Three Dimensions, Phys. Rev. Lett. 98, 106803 (2007).

[8] R. Roy, Topological phases and the quantum spin hall effect in three dimensions, Phys. Rev. B 79, 195322 (2009).

[9] M.Z. Hasan and C. L. Kane, Colloquium: topological insulators, Rev. Mod. Phys. 82, 3045 (2010).

[10] J. E. Moore, The birth of topological insulators, Nature (London) 464, 194 (2010).

[11] X.-L. Qi and S.-C. Zhang, Topological insulators and superconductors, Rev. Mod. Phys. 83, 1057 (2011).

[12] Y. Xia, D. Qian, D. Hsieh, L. Wray, A. Pal, H. Lin, A. Bansil, D. Grauer, Y. S. Hor, R. J. Cava, and M. Z. Hasan, Observation of a large-gap topological-insulator class with a single dirac cone on the surface, Nat. Phys. 5, 398 (2009).

[13] In this paper, the shorthand SPT, when it appears alone, always denotes a state in bosonic and spin systems unless otherwise specified, such as "fermionic SPT."

[14] F. D. M. Haldane, Nonlinear Field Theory of Large-Spin Heisenberg Antiferromagnets: Semiclassically Quantized Solitons of the One-Dimensional Easy-Axis Néel State, Phys. Rev. Lett. 50, 1153 (1983).

[15] F. D. M. Haldane, Continuum dynamics of the 1-D heisenberg antiferromagnet: Identification with the $O(3)$ nonlinear sigma model, Phys. Lett. A 93A, 464 (1983).

[16] I. Affleck and F. D. M. Haldane, Critical theory of quantum spin chains, Phys. Rev. B 36, 5291 (1987).

[17] I. Affleck, Quantum spin chains and the Haldane gap, J. Phys. Condens. Matter 1, 3047 (1989).

[18] F. Pollmann, A. M. Turner, E. Berg, and M. Oshikawa, Entanglement spectrum of a topological phase in one dimension, Phys. Rev. B 81, 064439 (2010).

[19] A. Kapustin, Symmetry protected topological phases, anomalies, and cobordisms: Beyond group cohomology, arXiv:1403.1467; Bosonic topological insulators and paramagnets: A view from cobordisms, arXiv:1404.6659.

[20] It should be pointed out that the relation between these different mathematical tools (i.e., group cohomology and cobordism) has been studied in Ref. [21], and those states that were "beyond group cohomology classification" now are still classified by a more generalized cohomology theory.

[21] X.-G. Wen, Construction of bosonic symmetry-protectedtrivial states and their topological invariants via $G \times S O(\infty)$ nonlinear $\sigma$ models, Phys. Rev. B 91, 205101 (2015).

[22] X.-G. Wen, Classifying gauge anomalies through symmetry-protected trivial orders and classifying gravitational anomalies through topological orders, Phys. Rev. D 88, 045013 (2013).

[23] M. Levin and Z. C. Gu, Braiding statistics approach to symmetry-protected topological phases, Phys. Rev. B 86, 115109 (2012).

[24] Y. M. Lu and A. Vishwanath, Theory and classification of interacting integer Topological phases in two dimensions: A Chern-Simons approach, Phys. Rev. B 86, 125119 (2012).

[25] T. Senthil and M. Levin, Integer Quantum Hall Effect for Bosons, Phys. Rev. Lett. 110, 046801 (2013).

[26] N. Regnault and T. Senthil, Microscopic model for the boson integer quantum hall effect, Phys. Rev. B 88, 161106 (2013).

[27] S. Furukawa and M. Ueda, Integer Quantum Hall State in Two-Component Bose Gases in a Synthetic Magnetic Field, Phys. Rev. Lett. 111, 090401 (2013).

[28] Y.-H. Wu and J. K. Jain, Quantum hall effect of twocomponent bosons at fractional and integral fillings, Phys. Rev. B 87, 245123 (2013).

[29] P. Ye and X.-G. Wen, Projective construction of twodimensional symmetry-protected topological phases with $U(1), S O(3)$, or $S U(2)$ symmetries, Phys. Rev. B 87, 195128 (2013).

[30] Y. M. Lu and D. H. Lee, Spin quantum hall effects in featureless nonfractionalized spin-1 magnets, Phys. Rev. B 89, 184417 (2014).

[31] P. Ye and J. Wang, Symmetry-protected topological phases with charge and spin symmetries: Response theory and dynamical gauge theory in two and three dimensions, Phys. Rev. B 88, 235109 (2013).

[32] M. Mulligan and M. P. A. Fisher, Partially split hall bar: Tunneling in the bosonic integer quantum hall effect, Phys. Rev. B 89, 205315 (2014).

[33] Z. X. Liu, J. W. Mei, P. Ye, and X.-G. Wen, U(1) × U(1) symmetry-protected topological order in Gutzwiller wave functions, Phys. Rev. B 90, 235146 (2014).

[34] M. Cheng and Z. C. Gu, Topological Response Theory of Abelian Symmetry-Protected Topological Phases in Two Dimensions, Phys. Rev. Lett. 112, 141602 (2014).

[35] J. Oon, G. Y. Cho, and C. Xu, Two-dimensional symmetryprotected topological phases with $P S U(N)$ and timereversal symmetry, Phys. Rev. B 88, 014425 (2013).

[36] O. M. Sule, X. Chen, and S. Ryu, Symmetry-protected topological phases and orbifolds: Generalized laughlin's argument, Phys. Rev. B 88, 075125 (2013).

[37] X.-G. Wen, Symmetry-protected topological invariants of symmetry-protected topological phases of interacting bosons and fermions, Phys. Rev. B 89, 035147 (2014).

[38] L. Y. Hung and Y. Wan, K-matrix construction of symmetry-enriched phases of matter, Phys. Rev. B 87, 195103 (2013). 
[39] Z. X. Liu and X. G. Wen, Symmetry-Protected Quantum Spin Hall Phases in Two Dimensions, Phys. Rev. Lett. 110, 067205 (2013).

[40] Z. Bi, A. Rasmussen, and C. Xu, Classification and description of bosonic symmetry protected topological phases with semiclassical nonlinear sigma models, Phys. Rev. B 91, 134404 (2015).

[41] Z.-X. Liu, Z.-C. Gu, and X.-G. Wen, Microscopic Realization of Two-Dimensional Bosonic Topological Insulators, Phys. Rev. Lett. 113, 267206 (2014).

[42] X. Chen, Y.-M. Lu, and A. Vishwanath, Symmetryprotected topological phases from decorated domain walls, Nat. Commun. 5, 3507 (2014).

[43] J. W. Mei and X.-G. Wen, Design local spin models for Gutzwiller-projected parton wave functions, arXiv:1407.0869.

[44] J. Wang, Z.C. Gu, and X.-G. Wen, Field-Theory Representation of Gauge-Gravity Symmetry-Protected Topological Invariants, Group Cohomology, and Beyond, Phys. Rev. Lett. 114, 031601 (2015).

[45] F. Burnell, X. Chen, L. Fidkowski, and A. Vishwanath, Exactly soluble model of a three-dimensional symmetryprotected topological phase of bosons with surface topological order, Phys. Rev. B 90, 245122 (2014).

[46] Z. Bi, A. Rasmussen, and C. Xu, Line defects in threedimensional symmetry-protected topological phases, Phys. Rev. B 89, 184424 (2014).

[47] A. Vishwanath and T. Senthil, Physics of ThreeDimensional Bosonic Topological Insulators: SurfaceDeconfined Criticality and Quantized Magnetoelectric Effect, Phys. Rev. X 3, 011016 (2013).

[48] C. Wang and T. Senthil, Boson topological insulators: A window into highly entangled quantum phases, Phys. Rev. B 87, 235122 (2013).

[49] P. Ye and X.-G. Wen, Constructing symmetric topological phases of bosons in three dimensions via fermionic projective construction and dyon condensation, Phys. Rev. B 89, 045127 (2014).

[50] C. Xu and T. Senthil, Wave functions of bosonic symmetry protected topological phases, Phys. Rev. B 87, 174412 (2013).

[51] Y. Wan, J. Wang, and H. He, Twisted gauge theory model of topological phases in three dimensions, arXiv:1409.3216.

[52] A. Mesaros and Y. Ran, Classification of symmetry enriched topological phases with exactly solvable models, Phys. Rev. B 87, 155115 (2013).

[53] S. Geraedts and O. Motrunich, Model Realization and Numerical Studies of a Three-Dimensional Bosonic Topological Insulator and Symmetry-Enriched Topological Phases, Phys. Rev. X 4, 041049 (2014).

[54] D. F. Mross, A. Essin, and J. Alicea, Composite Dirac Liquids: Parent States for Symmetric Surface Topological Order, Phys. Rev. X 5, 011011 (2015).

[55] By $\mathbb{Z}_{2} \times \mathbb{Z}_{2}$, we mean that a pair of $\mathbb{Z}_{2}$ indices can be defined and leads to $2 \times 2=4$ distinct phases labeled by $(0,0),(1,0),(0,1),(1,1)$. Among them, $(0,0)$ is a trivial SPT state, and $(1,0)$ and $(0,1)$ are two distinct root states. The last one, i.e., $(1,1)$, can be easily achieved by simply putting the two root states together in $3 \mathrm{D}$ space. This is the intuitive reason why $(1,0)$ and $(0,1)$ are called "root states."
[56] E. Witten, Dyons of charge $e \theta / 2 \pi$, Phys. Lett. 86B, 283 (1979).

[57] A. S. Goldhaber, R. MacKenzie, and F. Wilczek, Field corrections to induced statistics, Mod. Phys. Lett. A 04, 21 (1989).

[58] M. A. Metlitski, C. L. Kane, and M. P. A. Fisher, Bosonic topological insulator in three dimensions and the statistical witten effect, Phys. Rev. B 88, 035131 (2013).

[59] X.-L. Qi, T. L. Hughes, and S.-C. Zhang, Topological field theory of time-reversal invariant insulators, Phys. Rev. B 78, 195424 (2008).

[60] K. Walker and Z. Wang, $(3+1)$-TQFTs and topological insulators, Front. Phys. 7, 150 (2012).

[61] J. Cano, M. Cheng, M. Mulligan, C. Nayak, E. Plamadeala, and J. Yard, Bulk-edge correspondence in $(2+1)$-dimensional Abelian topological phases, Phys. Rev. B 89, 115116 (2014).

[62] E. Plamadeala, M. Mulligan, and C. Nayak, Short-range entangled bosonic states with chiral edge modes and $T$ duality of heterotic strings, Phys. Rev. B 88, 045131 (2013).

[63] X.-G. Wen, Quantum Field Theory of Many-Body Systems-From the Origin of Sound to an Origin of Light and Electrons (Oxford University Press, Oxford, 2004).

[64] B. Blok and X.-G. Wen, Effective theories of the fractional quantum hall effect: Hierarchy construction, Phys. Rev. B 42, 8145 (1990).

[65] N. Read, Excitation Structure of the Hierarchy Scheme in the Fractional Quantum Hall Effect, Phys. Rev. Lett. 65, 1502 (1990).

[66] J. Fröhlich and A. Zee, Large scale physics of the quantum hall fluid, Nucl. Phys. B364, 517 (1991).

[67] J. Fröhlich and T. Kerler, Universality in quantum hall systems, Nucl. Phys. B354, 369 (1991).

[68] X.-G. Wen and A. Zee, Classification of Abelian quantum hall states and matrix formulation of topological fluids, Phys. Rev. B 46, 2290 (1992).

[69] J. Fröhlich and U.M. Studer, Gauge invariance and current algebra in nonrelativistic many-body theory, Rev. Mod. Phys. 65, 733 (1993).

[70] X.-G. Wen, Topological orders and edge excitations in fractional quantum hall states, Adv. Phys. 44, 405 (1995).

[71] Here, for readers who are unfamiliar with the basic conventions" $\wedge$," we list some of them here. $d a \equiv f$, which is a 2 -form field strength of 1 -form gauge field $a$. Thus, $\int b \wedge d a=\int b \wedge f=\int d^{4} x\left(b_{\mu \nu} / 2\right)\left(f_{\lambda \rho} / 2\right) \epsilon^{\mu \nu \lambda \rho}=$ $\int d^{4} x \frac{1}{2} b_{\mu \nu} \partial_{\lambda} a_{\rho} \epsilon^{\mu \nu \lambda \rho} ; \int b \wedge b=\int d^{4} x\left(b_{\mu \nu} / 2\right)\left(b_{\lambda \rho} / 2\right) \epsilon^{\mu \nu \lambda \rho}=$ $\int d^{4} x \frac{1}{4} b_{\mu \nu} b_{\lambda \rho} \epsilon^{\mu \nu \lambda \rho} ; \int a \wedge d a=\int a \wedge f=\int d^{3} x a_{\mu}\left(f_{\nu \lambda} / 2\right) \epsilon^{\mu \nu \lambda}=$ $\int d^{3} x a_{\mu} \partial_{\nu} a_{\lambda} \epsilon^{\mu \nu \lambda}$.

[72] C. Dasgupta and B. I. Halperin, Phase Transition in a Lattice Model of Superconductivity, Phys. Rev. Lett. 47, 1556 (1981).

[73] D. R. Nelson, Vortex Entanglement in High- $T_{c}$ Superconductors, Phys. Rev. Lett. 60, 1973 (1988).

[74] M. P. A. Fisher and D. H. Lee, Correspondence between two-dimensional bosons and a bulk superconductor in a magnetic field, Phys. Rev. B 39, 2756 (1989).

[75] O. I. Motrunich and T. Senthil, Origin of artificial electrodynamics in three-dimensional bosonic models, Phys. Rev. B 71, 125102 (2005). 
[76] M. Franz, Vortex-boson duality in four space-time dimensions, Europhys. Lett. 77, 47005 (2007).

[77] B. Zwiebach, A First Course in String Theory (Cambridge University Press, Cambridge, England, 2009).

[78] S.-J. Rey, Higgs mechanism for Kalb-Ramond gauge field, Phys. Rev. D 40, 3396 (1989).

[79] X.-G. Wen, Vacuum degeneracy of chiral spin states in compactified space, Phys. Rev. B 40, 7387 (1989).

[80] X.-G. Wen and Q. Niu, Ground-state degeneracy of the fractional quantum hall states in the presence of a random potential and on high-genus Riemann surfaces, Phys. Rev. B 41, 9377 (1990).

[81] X.-G. Wen, Topological orders in rigid states, Int. J. Mod. Phys. B 04, 239 (1990).

[82] B. I. Halperin, Statistics of Quasiparticles and the Hierarchy of Fractional Quantized Hall States, Phys. Rev. Lett. 52, 1583 (1984).

[83] D. Arovas, J. R. Schrieffer, and F. Wilczek, Fractional Statistics and the Quantum Hall Effect, Phys. Rev. Lett. 53, 722 (1984).

[84] B. I. Halperin, Quantized hall conductance, currentcarrying edge states, and the existence of extended states in a two-dimensional disordered potential, Phys. Rev. B 25, 2185 (1982).

[85] X.-G. Wen, Theory of the edge states in fractional quantum hall effects, Int. J. Mod. Phys. B 06, 1711 (1992).

[86] M. Blau and G. Thompson, Topological gauge theories of antisymmetric tensor fields, Ann. Phys. (N.Y.) 205, 130 (1991).

[87] M. Bergeron, G. W. Semenoff, and R. J. Szabo, Canonical BF-Type topological field theory and fractional statistics of strings, Nucl. Phys. B437, 695 (1995).

[88] R. J. Szabo, String holonomy and extrinsic geometry in four-dimensional topological gauge theory, Nucl. Phys. B531, 525 (1998).

[89] G. T. Horowitz, Exactly soluble diffeomorphism invariant theories, Commun. Math. Phys. 125, 417 (1989).

[90] L. Smolin, Quantum gravity with a positive cosmological constant, arXiv:hep-th/0209079.

[91] In the standard definition of Cartan matrix [92], the offdiagonal entries should be nonpositive. However, we may prove that if a pair of off-diagonal entries of $\Lambda_{t 2}$ (e.g., $\Lambda^{12}=\Lambda^{21}=1$ ) changes sign (i.e., now $\Lambda^{12}=\Lambda^{21}=-1$ ), this sign change is nothing but a $\mathbb{G L}$ transformation. The same statement is also valid for the Cartan matrix of an SO (8) group.

[92] See http://mathworld.wolfram.com/CartanMatrix.html.

[93] A. Kitaev, Anyons in an exactly solved model and beyond, Ann. Phys. (Amsterdam) 321, 2 (2006).

[94] J. Milnor and D. Husemoller, Symmetric Bilinear Forms (Springer-Verlag, New York, 1973).

[95] R. J. Milgram, Surgery with coefficients, Ann. Math. 100, 194 (1974).

[96] A. Kitaev, Fault-tolerant quantum computation by anyons, Ann. Phys. (Amsterdam) 303, 2 (2003).

[97] M. Levin and A. Stern, Classification and analysis of twodimensional Abelian fractional topological insulators, Phys. Rev. B 86, 115131 (2012).

[98] R. Dijkgraaf and E. Witten, Topological gauge theories and group cohomology, Commun. Math. Phys. 129, 393 (1990).

[99] D. Gaiotto, A. Kapustin, N. Seiberg, and B. Willett, Generalized globalsymmetries, arXiv:1412.5148.

[100] P. Bonderson, C. Nayak, and X.-L. Qi, A time-reversal invariant topological phase at the surface of a $3 D$ topological insulator, J. Stat. Mech. (2013) P09016.

[101] C. Wang, A. C. Potter, and T. Senthil, Gapped symmetry preserving surface state for the electron topological insulator, Phys. Rev. B 88, 115137 (2013).

[102] X. Chen, L. Fidkowski, and A. Vishwanath, Symmetry enforced non-Abelian topological order at the surface of a topological insulator, Phys. Rev. B 89, 165132 (2014).

[103] M. A. Metlitski, C. L. Kane, and M. P. A. Fisher, A symmetry-respecting topologically-ordered surface phase of $3 d$ electron topological insulators, arXiv:1306.3286.

[104] G. Y. Cho and J. Moore, Topological BF field theory description of topological insulators, Ann. Phys. (Amsterdam) 326, 1515 (2011).

[105] A. Chan, T. L. Hughes, S. Ryu, and E. Fradkin, Effective field theories for topological insulators by functional bosonization, Phys. Rev. B 87, 085132 (2013).

[106] A. Chan, S. Ryu, and E. Fradkin (unpublished).

[107] Z. C. Gu, J. Wang, and X.-G. Wen, Multi-kink topological terms and charge-binding domain-wall condensation induced symmetry-protected topological states: Beyond Chern-Simons/BF theory, arXiv:1503.01768v1. 